\title{
Fluid Approximation of Smart Grid Systems: Optimal Control of Energy Storage Units
}

\author{
by \\ Rasha Ibrahim Sakr, B.Sc. \\ A thesis submitted to the \\ Faculty of Graduate and Postdoctoral Affairs \\ in partial fulfillment of the requirements for the degree of
}

Master of Applied Science in Mathematics

School of Mathematics and Statistics

Carleton University

Ottawa, Ontario

January 2015

(C) Copyright

Rasha Ibrahim Sakr, 2015 


\section{Abstract}

A model of the smart grid system with two different energy sources - the main grid and the energy storage units - is considered. The arriving power demands can be activated by either type of energy sources, with differing rates and costs. Finding an optimal policy that minimizes the expected long-run operational cost of the system is the main interest of this work.

The problem is considered in a so called heavy traffic regime, and is solved using fluid approximation techniques. The formal scaling limit of the problem leads to a simple deterministic optimization problem, whose solution is shown to be an achievable lower bound on the limiting cost of the stochastic problem. Three different scenarios are considered according to whether the batteries are disposable or rechargeable and whether the arrival rates are homogeneous or nonhomogeneous. The solution method provides a good alternative to numerical methods such as Markov Decision Processes. 


$$
\begin{gathered}
\text { To my kids, } \\
\text { Yumna, Suhayb, and Asmaa. }
\end{gathered}
$$




\section{Acknowledgments}

All praise is due to God, Most Gracious, Most Merciful, Whose bounties and blessings are ever dominating throughout my life.

I would like to express my deepest gratitude and appreciation to my supervisor, Prof. Gennady Shaikhet. Prof. Shaikhet has always been an invaluable source of support, guidance, and encouragement. I would also like to thank him for his devoted time and effort in making this thesis possible.

My deep gratitude to my mother, Dr. Hamida Attia, and my sisters for their unconditional support, unwavering confidence in me, and ever continuous prayers.

Finally, I would like to thank my husband, Dr. Muhammad Nummer, who shared with me every day throughout the course of this work. His support, patience, and understanding played a major role in helping me finish this thesis. 


\section{Table of Contents}

$\begin{array}{ll}\text { Abstract ii } & \text { ii }\end{array}$

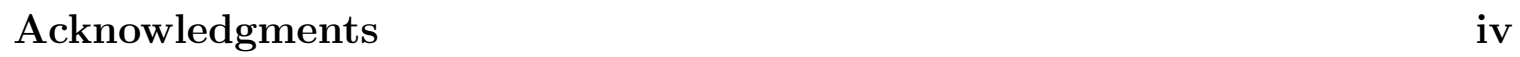

$\begin{array}{ll}\text { Table of Contents } & \text { v }\end{array}$

List of Figures viii

List of Acronyms $\quad \mathrm{x}$

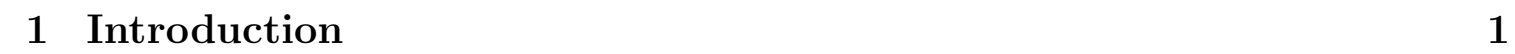

1.1 Motivation . . . . . . . . . . . . . . . . . . . . . . . 1

1.2 Goals and Contributions . . . . . . . . . . . . . . . . . . . 2

\begin{tabular}{|ll|}
\hline 2 & Background \\
\hline
\end{tabular}

2.1 Solving the Optimal Control Problems by Numerical Methods . . . . 5

2.1.1 The Electrical Vehicles (EV/PHEV) Charging Stations . . . . 6

2.1 .2 The Smart Power Grid . . . . . . . . . . . . . . . . . . . . . . 8

2.2 Solving the Optimal Control Problems by Fluid Approximation . . . 10

2.3 An Overview of the $M / M / N$ Queuing Model and its Fluid Model . . 11

2.3 .1 Dynamical Representation of the Model . . . . . . . . . . . . 12

2.3.2 The Fluid Approximation of the $M / M / N$ Queueing Model . . 13

2.3.3 Limit Theorem for the $M / M / N$ Queueing Model . . . . . . . 14

$2.3 .4 \quad$ Limit Theorem for the $M / M / \infty$ Queueing Model . . . . . . . 15 


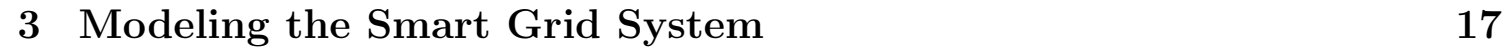

3.1 The Smart Grid System Without Batteries . . . . . . . . . . . . . . . 17

3.2 The DES Model of the Smart Grid System with Batteries. . . . . . . . 18

3.3 Heavy Loads and Optimal Control Problem of the DES Model . . . . 19

3.4 Fluid View of the DES Model . . . . . . . . . . . . . . . . . . . . . . 20

4 Solving The Optimal Control Problem of the DES Model 23

4.1 The Deterministic Optimal Control Problem of the DES Model . . . 23

4.1 .1 Part 1 of Solution. The Lower Bound on the Cost . . . . . . 24

4.1.2 Part 2 of Solution. The Optimal Policy for the DES Model . . 26

4.2 Stochastic Model. Interpretation of the Policy . . . . . . . . . . . . . 27

\begin{tabular}{|ll|}
5 & Generalizations of the Smart Grid System \\
\hline
\end{tabular}

5.1 The N-DES Model of the Smart Grid System . . . . . . . . . . . . . 30

5.2 The RES Model of the Smart Grid System . . . . . . . . . . . . . . . 32

5.3 Heavy Loads and Optimal Control Problem of the RES Model . . . . 33

5.4 Fluid View of the RES Model . . . . . . . . . . . . . . . . . . . . . . 34

5.4 .1 RES Model. Fluid Optimization . . . . . . . . . . . . . . . 36

5.4 .2 The Optimal Policy of the RES Model . . . . . . . . . . . . . 36

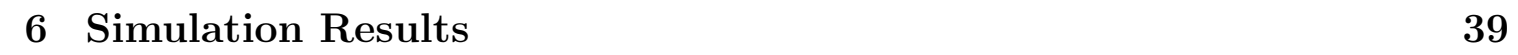

$6.1 \quad$ Simulation Results of $M / M / N$ Queuing Model . . . . . . . . . . . . . 39

6.2 Simulation Results of the DES Model . . . . . . . . . . . . . . . . . . 40

6.3 Simulation Results of the N-DES Model . . . . . . . . . . . . . . . . 42

6.4 Simulation Results of the RES Model . . . . . . . . . . . . . . . . . . 44

6.5 More Simulation Results of the Smart Grid . . . . . . . . . . . . . . . 46

\begin{tabular}{|lll}
\hline 7 & Conclusion and Future Work & 48
\end{tabular}

\begin{tabular}{ll}
\hline List of References & 49
\end{tabular}

\begin{tabular}{|ll}
\hline Appendix A Some Useful Theorems & 51
\end{tabular} 
\begin{tabular}{|ll}
\hline Appendix B Code for Simulations & 52
\end{tabular}

B.1 Simulation Programs of $M / M / N$ Queuing Model $\ldots \ldots \ldots \ldots . . . \quad 52$

B.2 Simulation Programs of DES Model . . . . . . . . . . . . . . 55

B.3 Simulation Programs of the N-DES Model … . . . . . . . . . 62

B.4 Simulation Programs of RES Model . . . . . . . . . . . . . . . . . . 69 


\section{List of Figures}

$2.1 \quad$ Smart vehicles' charging station with batteries $\ldots \ldots \ldots \ldots$

$2.2 \quad$ All possible transient states of the controlled MDP $\ldots \ldots \ldots . \quad 8$

2.3 The smart grid with rechargeable batteries [1] $\ldots \ldots \ldots \ldots$

2.4 The dynamics of $\mathcal{I}$ classes of customers, n-servers with abandonment system $[2] \ldots \ldots \ldots \ldots \ldots$

$2.5 \quad$ Continuous-time Markov chain of $M / M / N$ queueing model . . . . . . 11

$2.6 \quad$ Departure process of $M / M / 3$ queueing system $\ldots \ldots \ldots \ldots \ldots \ldots$

2.7 Deterministic view of service systems by fluid approximation. . . . . . 14

3.1 Continuous-time Markov chain of the smart grid without batteries . . 18

3.2 Dynamics of the proposed DES model $\ldots \ldots \ldots \ldots$

3.3 Continuous-time Markov chain of the proposed DES model . . . . . . 19

4.1 Dynamics of the proposed DES model given the control policy $h_{1} *$. . 28

4.2 Continuous-time Markov chain of the proposed DES model given the control policy $h_{1} * \ldots \ldots \ldots \ldots \ldots$

$5.1 \quad$ Dynamics of the proposed RES model . . . . . . . . . . . . . 32

5.2 Continuous-time Markov chain of the proposed RES model . . . . . . 33

$6.1 \quad$ A comparison of one sample path of $\bar{Q}^{n}(t)$ to fluid $Q(t)$ of the $M / M / N$ queuing model . . . . . . . . . . . . . . . . . . . . . 40

$6.2 \quad$ A comparison of one sample path of $\bar{X}_{1}^{n}(t)$ to fluid $X_{1}(t)$ of the DES model . . . . . . . . . . . . . . . . . . . . . . . 41

$\begin{array}{lll}6.3 & \text { A comparison of one sample path and the fluid } \mathcal{P}_{1}^{*} \text { of the DES model } & 42\end{array}$

$6.4 \quad$ A comparison of one sample path of $\bar{X}_{1}^{n}(t)$ to fluid $X_{1}(t)$ of the N-DES model . . . . . . . . . . . . . . . . . . . . . . 43 
$6.5 \quad$ A comparison of one sample path and the fluid $\mathcal{P}_{1}^{*}$ of the N-DES model 44

6.6 A comparison of one sample path of $\bar{X}_{2}^{n}(t)$ to fluid $X_{2}(t)$ of the RES model . . . . . . . . . . . . . . . . . . . . . . . 45

$6.7 \quad$ A comparison of one sample path and the fluid $\mathcal{P}_{2}^{*}$ of the RES model $\quad 46$

$6.8 \quad$ A comparison of the cost $\mathcal{P}_{1}^{h_{1}^{*}} \ldots \ldots \ldots \ldots \ldots 4$

$6.9 \quad$ A comparison of the cost $\mathcal{P}_{2}^{h_{2}^{*}} \ldots \ldots \ldots \ldots \ldots \ldots$ 


\section{List of Acronyms}

DES model The smart grid system in which disposable energy storage units (batteries) are used.

N-DES model The smart grid system in which disposable energy storage units (batteries) are used and the power demands are assumed to be non homogenous arrivals.

RES model The smart grid system in which rechargeable energy storage units (batteries) are used. 


\section{Chapter 1}

\section{Introduction}

\subsection{Motivation}

An electric grid is a network of power providers and consumers connected by transmission and distribution lines and operated by one or more control centres. A class of technologies are being developed with the goal of moving the utility electricity delivery systems towards a new digital future leading to what is commonly being referred to as "the smart grid". Such technologies include using computer-based remote control and automation. These digital intelligent systems are made possible by two-way communication technology and computer processing that has been used for decades in other industries [3]. Smart grids expand the capabilities of the electricity system to provide even greater benefits to customers such as increased efficiency in power utilization, reduced cost, and improved quality. They allow for better communication, automation, and coordination among power suppliers, consumers, and corresponding transportation and distribution networks [4].

Improving the grid requires different kinds of renewable energy sources and energy storage components (batteries). Batteries have become key components of the smart grid. They play a critical rule in sustaining the power grid especially at peak times [1]. In general, the main idea behind the use of batteries is to satisfy customers' demand without straining the grid. The required power demands can be satisfied by the power utility or by the local batteries. Excess power from the grid can be used to recharge batteries which can be used later to activate some of the power demands that exceed 
the maximum capacity of the grid.

There is a large body of literature on leveraging stored energy in various contexts. For example, in the electric charging stations, the use of batteries was proposed as a solution to manage the high load on the grid due to charging the increasing number of smart electric vehicles [4]. Although batteries are very expensive, they can be quite advantageous for electric utility operators and consumers if used with appropriate storage management policies. Bayram [4] addresses the problem of controlling the size, pricing, and cost parameters of batteries and develops a queuing model for charging stations employing batteries. The net profit is then expressed as a function of the revenue achieved due to charging vehicles and the cost related to batteries as well as the blocked customers. Various combinations of those variables are examined and allowable ranges for each of them are determined.

Koutsopoulos [1] discusses the need for using batteries in the smart grid with appropriate management policy. It formulates the total operational cost from the point of view of the utility operator as a convex function of the total power demands given certain policy. In addition, it offers a numerical solution using dynamic programming. Other researchers have explored optimizing the use of batteries in other areas such as in data centers and wireless networks [5, 6]. Atar [2] solves a control problem for multi class, many-servers with abandonment queuing network under fluid scaling and heavy traffic conditions. These conditions turned a stochastic control problem of such large and complicated system into a deterministic problem which is simple to solve.

Same method is applied in many different control problems such as in [7]. A rigours solution of demand load management and optimal control problem of batteries used in large and complicated systems like the smart grid remains a challenging research area.

\subsection{Goals and Contributions}

In the context of the smart grid, this thesis presents the use of the energy storage units (batteries) as a recipe to manage the load on the grid. A control problem of the number of batteries is considered and an easy asymptotic solution is proposed so that 
the total operational cost faced by the utility operator is minimized. The smart grid system is analyzed and the appropriate control policy of using batteries is determined for three different models. The first model is called the DES model which refers to the smart grid system in which disposable energy storage units (batteries) are used. This model has one customer class, a large number of servers (as the grid is expected to activate all the coming demands), and a number of backup servers. The customer class represents "power demands/tasks" that arrive according to Poisson process and have identical power requirements. The backup servers are the "disposable batteries". All service times whether by the grid or by batteries are independent and exponential random variables. Empty batteries get replaced with new ones consistently. Since all customers are going to be served upon arrival, there will be no waiting. Given the assumption that there are no missing customers due to blocking or abandonment, the total operational cost faced by the utility operator can be modeled as a function of the total number of power demands which received service from the grid as well as the number of customers which received service from batteries. Instead of solving the stochastic optimization problem first, a simple deterministic problem for DES model is developed and solved by fluid approximation. It is important to note that this approximation was possible by considering a sequence of many smart grid systems and allowing the arrival rate to be very big. The solution to this problem is shown to be asymptotically converging to the optimal solution.

The other two proposed models of the smart grid are called the N-DES model and the RES model. The N-DES model refers to the smart grid system in which disposable energy storage units (batteries) are used and the power demands are assumed to be non homogenous arrivals. The RES model refers to the smart grid system in which rechargeable energy storage units (batteries) are used. The total operational cost in case of using rechargeable batteries is modeled as a function of the total number of power demands which received service from the grid, the number of empty batteries that got recharged by grid, as well as the number of customers who received service from batteries.

To summarize, the main result of this work is that an interesting feature of fluid approximation help to obtain a simple control policy. Such policy is found to be 
asymptotically optimal, in the sense that it attains the asymptotic lower bound. The result of the homogenous arrivals case is first established, then the proof of a more general case where arrivals happen according to a nonhomogenous Poisson process is extended in addition to the RES model. 


\section{Chapter 2}

\section{Background}

This chapter is a brief review of relevant literature on solving the optimal control problem that minimizes the total operational cost associated with demand load management like in electrical vehicles $(\mathrm{EV} / \mathrm{PHEVs})$ charging stations and smart power grid. The chapter also discusses relevant work on using the fluid approximation method to find the optimal routing policy of jobs of different classes to a pool of servers that arises in many-servers queueing system with abandonment. Although this problem arises in systems like call centers, the solution by fluid approximation can be applied to find the optimal control policy of the capacity of batteries of the smart grid as this work will show. The reminder of the chapter provides an in depth review of the many-servers queueing system and its fluid approximation. Additionally, the fluid approximation of $M / M / \infty$ model is proved.

\subsection{Solving the Optimal Control Problems by Nu- merical Methods}

This section surveys work on different methods of solving the optimal control problem in the electrical vehicles $(\mathrm{EV} / \mathrm{PHEVs})$ charging stations and the smart power grid. The increased interest in the electrical vehicles will affect the electrical grid specially at the time of charging a large number of them at the same time. The smart power grid will rely on information and communication technologies and advanced control 
methods to manage the dynamic demand load and to ensure efficient use of electric energy. The main idea of the demand load control is to alleviate high demand load at peak times. In both systems batteries are used to manage the demand load. Batteries are very expensive, so their number is in need of management in order to minimize the total operational cost incurred by the utility operator. The time to charge or discharge batteries is in need of management as well.

\subsubsection{The Electrical Vehicles (EV/PHEV) Charging Stations}

Bayram [4] proposes a structure of the EV/PHEV charging stations where batteries can be used as backup devices. This structure is illustrated in Figure 2.1.

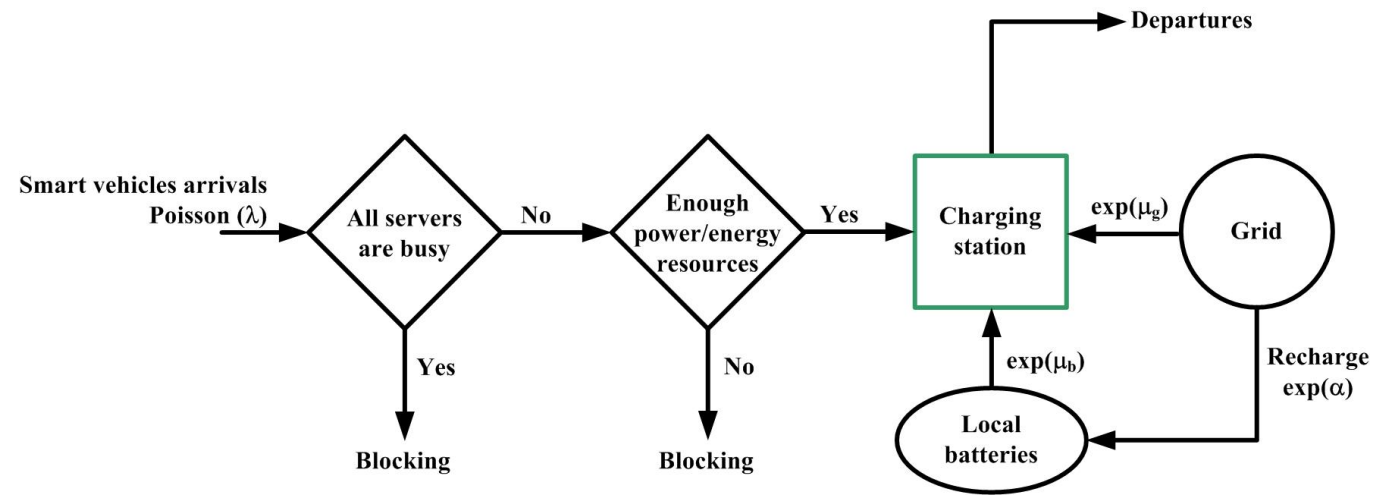

Figure 2.1: Smart vehicles' charging station with batteries

In the suggested model by Bayram [4], power demands arrive according to Poisson process with rate $\lambda>0$. Arriving vehicles can be served either by the grid or batteries. The grid service times are IID exponential random variables with rate $\mu_{g}>0$. Service times of batteries are also IID exponential random variables with rate $\mu_{b}>0$. The charging station has a finite capacity which can be considered as the maximum number of vehicles that can be charged simultaneously by the grid and batteries. At off-peak times, the empty batteries can be recharged by the grid at another rate $\alpha>0$. Also, vehicles that find the system busy and/or the energy level of batteries not enough get blocked. Thus, Bayram [4] models the system as Erlang-B system. The dynamics of this system are captured by a continuous-time birth-death Markov chain with finite two-dimensional state space, with one dimension corresponding to 
the number of vehicles that are being served in the system and the second dimension corresponding to the level of batteries.

Furthermore, Bayram [4] presents a model of the net profit that relates pricing parameters to the queuing model parameters such as the maximum number of vehicles that can simultaneously get charged by the grid (grid capacity), the max number of vehicles that can simultaneously get charged by batteries (the capacity of batteries), and the arrival rate. The net profit is set as the difference between the total revenue due to successfully charged vehicles either by grid or batteries and the total cost due to the operational cost of using the grid and batteries (installation and acquisition cost) and the cost due to blocked vehicles (dissatisfaction cost). Using simulation experiments, Bayram [4] explores the different combinations of all parameters in order to maximize the net profit, reduce the total cost, and achieve the maximum allowable arrival rate.

Karbasioun [8] models the electrical vehicles (EV/PHEV) charging stations differently. In his model, the capacity of the grid and batteries are assumed to be fixed deterministic parameters. Karbasioun [8] assumes that the price of the electrical power unit is determined by a Real Time Pricing (RTP) scheme. In this scheme, the price of the electrical power is continuously updated with respect to the state of the grid at each time instant. At each time $t$, the state of the charging station is determined by a three-dimensional state space, with one dimension corresponding to $i=i(t), i$ denotes the total number of vehicles being charged in the system at time $t, j=j(t), j$ denotes the level of the battery at time $t$, and $c$ denotes the price of each unit of electrical power in the grid. At each state and based on the three control actions, the next state is determined. The control actions are: the blocking probability of new arrivals, the charging rate of the battery, and the proportion of vehicles being served by battery. The out flow of the state $(\mathrm{c}, \mathrm{i}, \mathrm{j})$ is shown in Fig 2.2 . 


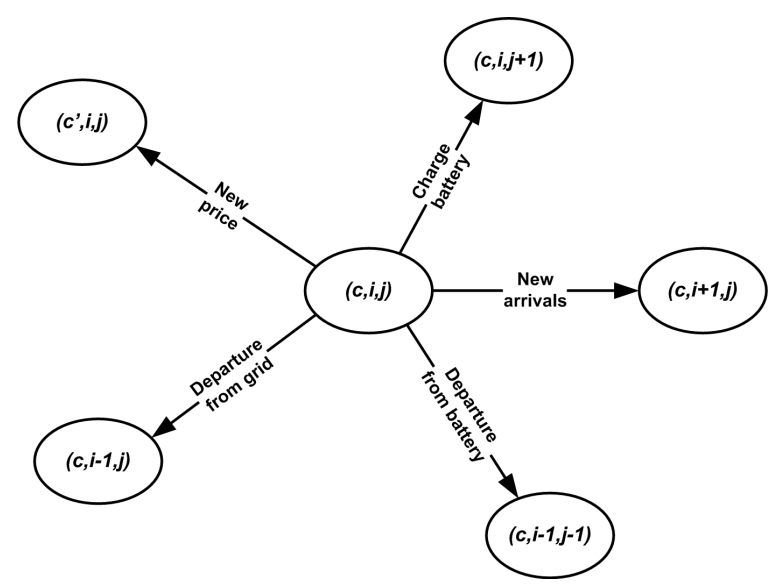

Figure 2.2: All possible transient states of the controlled MDP

The instantaneous cost function of the model presented by Karbasioun [8] is a function of the current state, the selected action, and the total revenue of the station at time $t$. This control problem is defined as Markov Decision Process (MDP). An MDP is a controlled stochastic process that satisfies the Markov property with costs associated to each state transition. A Markov decision problem consists of an MDP and a performance criterion. An MDP is described by four types of information, State space, decision set, transition probabilities, and expected rewards [9]. A solution to a Markov decision problem is called a policy, which determines the optimal control actions according to the performance criterion. Karbasioun [8] solves the MDP in which the controls are applied at transition instants so that an appropriate cost is optimized. The optimal policy is obtained both for the finite and infinite horizon problems. Karbasioun [8] shows that the optimal policy is stationary and hence depends only on the current state.

\subsubsection{The Smart Power Grid}

Koutsopoulos [1] emphasizes the importance of using batteries for demand load management in the smart grid. It addresses the problem of finding the optimal control policy of batteries for the smart grid such that the total operational cost incurred by the utility operator over a time horizon is minimized. The cost model defined by [1], $\mathcal{P}(t)$, is a convex function of the total consumed power on the grid at time $t$. This 
power is estimated as the sum of active demands that are served by the grid, plus the charging rate of batteries, $\alpha(t)$. The cost reflects that each unit of additional power needed to satisfy the increasing demand becomes more expensive. In the online problem, the paper divides the time into slots of unit interval over which the expected total operational cost is minimized. At each time slot $t$, the energy storage unit with capacity $B(t)$ may be charging or discharging.

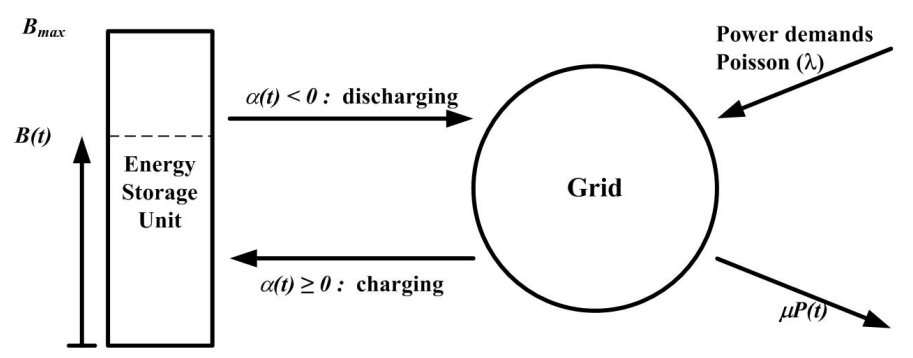

Figure 2.3: The smart grid with rechargeable batteries 1

The control problem of [1] is formulated as a discrete-time Markov decision process. The decision variable $\alpha(t)$ is defined as the rate at which the grid recharges or discharges batteries. If batteries are discharged (i.e $\alpha(t)<0$ ), part of the power demand on the grid at time $t$ will be served from batteries. On the other hand, if batteries are charged (i.e $\alpha(t) \geq 0$ ), the energy will flow into batteries and the total grid consumption will be increased by $\alpha(t)$. The dynamics of the model are shown in Figure 2.3. A threshold control policy is also derived and defined as follows:

1. If the power demands activated by the grid are less than the threshold, charge batteries with rate $\alpha(t)$ equal to the difference.

2. If the power demands activated by the grid are greater than the threshold, discharge batteries with rate $\alpha(t)$ equal to the difference.

The threshold is set to be the maximum number of power demands that can be activated simultaneously by the grid. This policy is obtained for both finite and infinite horizon problem.

Solving the optimal control problem of batteries of the smart grid as an MDP will be difficult due to computations and complexity of such a large system. Instead, it is decided in this work to use the fluid approximation method. 


\subsection{Solving the Optimal Control Problems by Fluid Approximation}

This section is a review of a relevant work on solving the optimal control problems by fluid approximation method. Atar [2] solves the optimal routing policy problem of many customer classes and many servers system. In his model, there is a pool of independent $n$ servers with identical capabilities that cater to the customers. Additionally, there are $\mathcal{I}$ classes of customers arriving according to Poisson processes with rates that are proportional to $n, \lambda_{i}^{n}$, where $\lambda_{i}^{n}>0$ and $i \in\{1, \ldots, I\}$. Service times are IID exponential random variables with rates $\mu_{i}>0$. Customers who find the system busy upon arrival join a queue of their own class. While waiting in queue, customers may abandon the system with rates $\theta_{i}>0$. Once any of the busy servers becomes free, it has to decide which of the waiting customers to accept to service. This is illustrated in Figure 2.4.

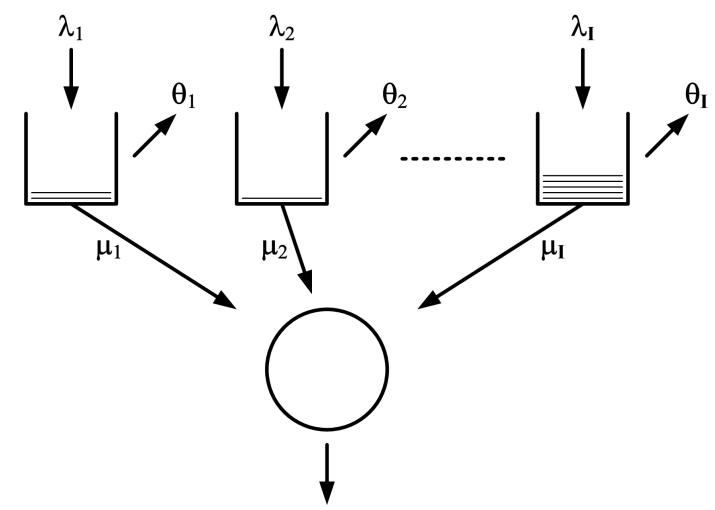

Figure 2.4: The dynamics of $\mathcal{I}$ classes of customers, n-servers with abandonment system [2]

A stochastic control problem arises by considering the long run average cost due to holding and reneging, in which the total cost per unit time for class-i customer is a given constant, $c_{i}$. The control involves dynamic routing of jobs of different classes to the server pool. The problem is in the fully exponential setting and under overloaded conditions (when the incoming service requirement strictly exceeds the service capacity, as other wise all queues will be empty in steady state under any 
non-idling service scheme). Hence, Atar 2] instead studies this problem in a many server fluid regime. In this regime the hard stochastic control problem can be shown easily to be equivalent to a deterministic optimization problem that is simpler to solve. The control policy is given as a simple priority policy that assigns strict priority to classes according to the order of their indices $c_{i} \mu_{i} / \theta_{i}$.

\subsection{An Overview of the $M / M / N$ Queuing Model and its Fluid Model}

This section introduces the $M / M / N$ queuing model and proves its fluid approximation. Then, this case is used to prove the fluid approximation of $M / M / \infty$ model that will serve as the basis for much of the work presented next in this thesis.

Consider an $M / M / N$ queuing model which has an infinite capacity. This queueing model is a Markov jump process as shown in Figure 2.5.

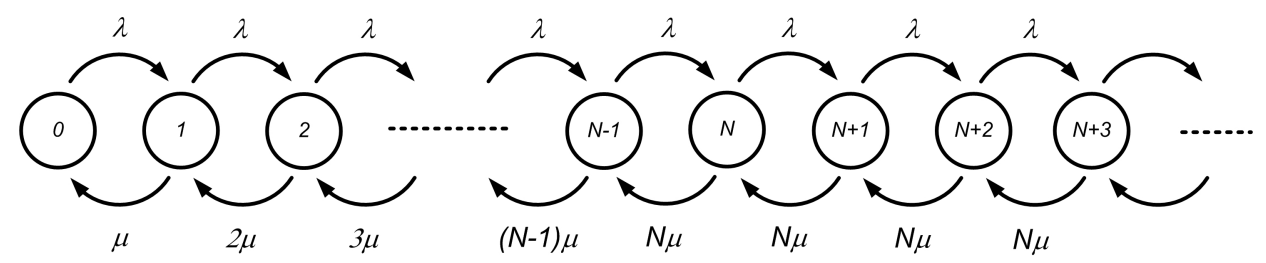

Figure 2.5: Continuous-time Markov chain of $M / M / N$ queueing model

Where it can be used to analyze service systems in which customers arrive according to Poisson process with rate $\lambda>0$. In such systems, there are $N$ identical servers whose service times are exponential random variables with rate $\mu>0$. New arrival to the system gets immediately served if there is at least one free server. Otherwise, the customer joins the queue. The customer departs the system after being served and the next customer in line, if there is any, gets service at the time when one of the busy servers becomes free 10 . 


\subsubsection{Dynamical Representation of the Model}

In order to continue, an alternative description of the dynamics of the $M / M / N$ model is introduced. Let $A(t)$ be the total number of customers that have arrived during the time interval $[0, t]$ and let $Q(t)$ be the total number of customers in the system at time $t$ (in queue plus in service). Let $D(t)$ be the total number of customers that finish service during the time interval $(0, t]$. As proposed in [11], the number of departures by time $t\{D(t), t \geq 0\}$ can be expressed as $D(t)=S\left(\sum_{i=1}^{N} B_{i}(t)\right)$, where $S(t)$ is Poisson process that counts the total number of departures in the interval $[0, t]$ with rate $\mu>0$, and $B_{i}(t)$ is the busy time of server $i$.
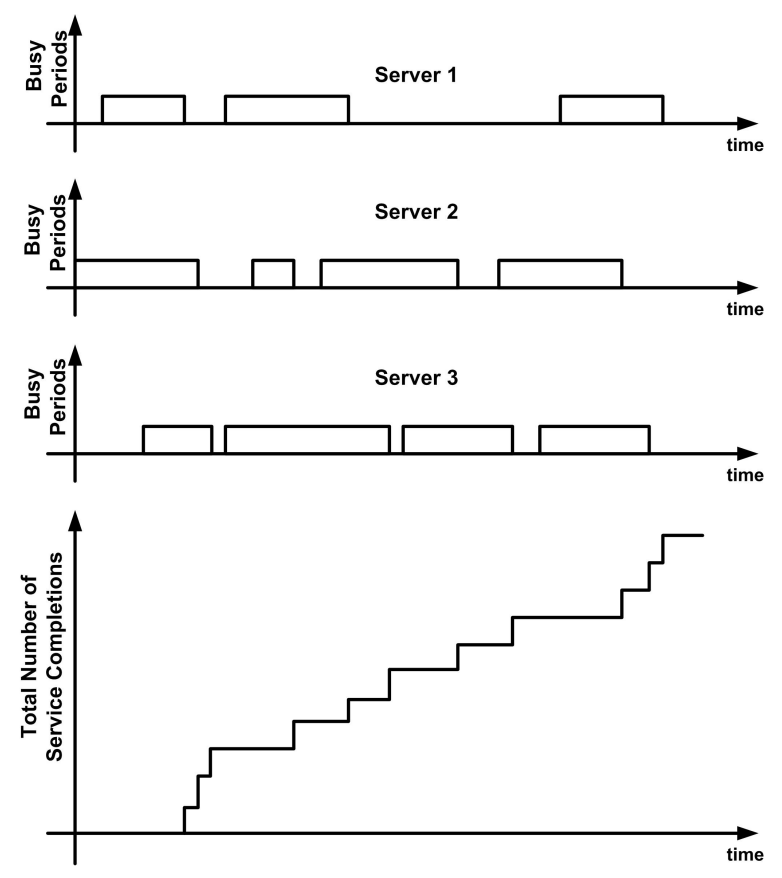

Figure 2.6: Departure process of $M / M / 3$ queueing system

The total busy time for all servers can be computed as follows:

$$
\sum_{i=1}^{N} B_{i}(t)=\sum_{i=1}^{N}\left(\int_{0}^{t} 1_{\text {server i is busy at time } s} d s\right)
$$

where $\left\{1_{\text {server } i \text { is busy at times } s}\right\}$ is the indicator function that is equal to 1 whenever server $i$ is busy. Then, the departure process of this queueing model can be represented alternatively by 


$$
D(t)=S\left(\int_{s=0}^{t}(Q(s) \wedge N) d s\right),
$$

where $a \wedge b=\min (a, b)$. An example of a departure process of $M / M / 3$ is shown in Figure 2.6 for illustration. Assume that the initial total number of customers in the system, $Q(0)$, is given. $Q(t)$, must satisfy the relation

$$
Q(t)=Q(0)+A(t)-D(t)
$$

From Equation (2.2),

$$
Q(t)=Q(0)+A(t)-S\left(\int_{s=0}^{t}(Q(s) \wedge N) d s\right)
$$

\subsubsection{The Fluid Approximation of the $M / M / N$ Queueing Model}

Consider a sequence of $M / M / N$ systems parameterized by $n, n \rightarrow \infty$, (emphasized by using the superscript ${ }^{n}$ ) and assume that the arrival rate of the customers is proportional to $n$, that is $\lambda^{n}=\lambda n$ for some $\lambda>0$ and assume $N^{n}=n$. The fluid approximation of such system is concerned primarily with the behaviour of the limits of the scaled processes $A^{n}(t) / n, Q^{n}(t) / n$, and $D^{n}(t) / n$ as $n \rightarrow \infty[12$. For any process $f$, denote the scaled process in the heavy load conditions, $\bar{f}^{n}=f / n$. The pre-limit scaled process of the total number of jobs in the system can be given by

$$
\bar{Q}^{n}(t)=\bar{Q}^{n}(0)+\bar{A}^{n}(t)-\bar{S}^{n}\left(n \int_{0}^{t}\left(\bar{Q}^{n}(s) \wedge \bar{N}^{n}\right) d s\right) .
$$

The Poisson processes, $\bar{A}^{n}(t)$ and $\bar{S}^{n}(n t)$ converges weakly to $\lambda t$ and $\mu t$ respectively. See the definition of weak convergence given by [13]. Hence, the convergence of $\bar{Q}^{n}$ to the limiting queue-length $\bar{Q}$ can be given by

$$
\bar{Q}(t)=\bar{Q}(0)+\lambda t-\mu \int_{s=0}^{t}(\bar{Q}(s) \wedge 1) d s
$$


Equation (2.6) implies that the fluid approximation limit of the N-server queue can be interpreted as the fluid level of a deterministic, continuous flow system. Such system would have a single buffer, with fluid continuously flowing in at a constant rate $\lambda$ and flowing out at a constant rate $\mu$. (see Figure 2.7). Therefore, one can refer to this fluid approximation limit as the fluid limit. A detailed example of an $M / M / N$ queuing model and simulation results are given in Chapter 6 to prove the fluid approximation given by Equation (2.6).

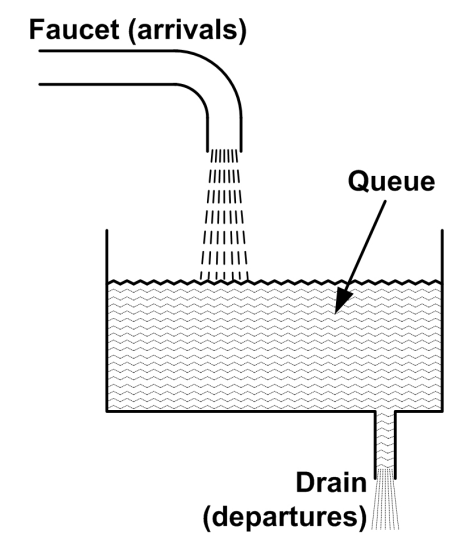

Figure 2.7: Deterministic view of service systems by fluid approximation.

\subsubsection{Limit Theorem for the $M / M / N$ Queueing Model}

The Stone's theorem [13] can also be used to prove the fluid approximation for $M / M / N$ queuing model given by Equation (2.6). The proof is to show that, in the limit, the scaled infinitesimal mean of the process $\bar{Q}^{n}$ converges to the infinitesimal mean of the limiting queue-length $\bar{Q}$.

First, the infinitesimal mean of the process $Q^{n}(t)$ is calculated. Since $Q^{n}(t)$ is a Markov process, $E\left(Q^{n}(t+h)-Q^{n}(t) \mid Q^{n}(t)\right)=m_{Q^{n}(t)} h+o(h)$, and

$$
m_{Q^{n}(t)}=\lim _{h \rightarrow 0} \frac{1}{h} E\left(Q^{n}(t+h)-Q^{n}(t) \mid Q^{n}(t)\right) .
$$

Additionally, since arrivals and departures are Poisson processes with instantaneous 
rates $\lambda^{n}$ and $\min \left(Q^{n}(t), N^{n}\right) \mu$, the infinitesimal mean can be given by

$$
m_{Q^{n}(t)}=\lambda^{n}-\min \left(N^{n}, Q^{n}(t)\right) \mu
$$

As a result, for the scaled process $m_{\bar{Q}^{n}(t)}$, one can have

$$
\frac{1}{n} m_{Q(t)}=\frac{\lambda^{n}}{n}-\min \left(\frac{N^{n}}{n}, \frac{Q^{n}(t)}{n}\right) \mu=\lambda-\min \left(\bar{Q}^{n}(t), 1\right) \mu
$$

As for the infinitesimal variance of the process $\bar{Q}^{n}$ one can first have

$$
v_{Q^{n}(t)}=\lim _{h \rightarrow 0} \frac{1}{h} E\left(\left(Q^{n}(t+h)-Q^{n}(t)\right)^{2} \mid Q^{n}(t)\right)
$$

Implying,

$$
\left.v_{Q^{n}(t)}=\lambda^{n}+\min \left(N^{n}, Q^{n}(t)\right) \mu\right)
$$

As $n \rightarrow \infty$ however, the infinitesimal variance of the scaled process $\bar{Q}^{n}$ converges to zero, 1.e

$$
v_{\bar{Q}^{n}(t)}=\lim _{n \rightarrow \infty} \frac{1}{n^{2}} v_{Q^{n}(t)}=0 .
$$

This concludes the proof of the convergence of $\bar{Q}^{n}$ to $\bar{Q}$ as $n \rightarrow \infty$.

\subsubsection{Limit Theorem for the $M / M / \infty$ Queueing Model}

In a similar fashion one can treat the $M / M / \infty$ systems, which are called insensitive systems as there is always a free server ready to serve new arrival customers and there are no queues at all. Each customer does not wait in line to get served, but spends a time in the system equal to the service time only [14]. Assume the same heavy load setting $\lambda^{n}=\lambda n$. Let $X^{n}(t)$ be the total number of customers in the system at time $t$ which is equal to the number of busy servers. In this case the infinitesimal mean of the scaled process $\bar{X}^{n}$ can be given by

$$
m_{\bar{X}^{n}(t)}=\frac{\lambda^{n}}{n}-\left(\frac{X^{n}(t)}{n}\right) \mu=\lambda-\bar{X}^{n}(t) .
$$


With the infinitesimal variance once again converging to zero, the above will imply the convergence as $n \rightarrow \infty$ of $\bar{X}^{n}$ to a deterministic process $\bar{X}$, satisfying

$$
\bar{X}(t)=\bar{X}(0)+\lambda t-\mu \int_{s=0}^{t} \bar{X}(s) d s
$$




\section{Chapter 3}

\section{Modeling the Smart Grid System}

This chapter presents the DES model of the smart grid system. The core idea of this model is that power demands can be served either by constant power supplied by the utility (grid) or by disposable batteries. Empty batteries get replaced by new ones which in turn are used to support temporary high loads on the grid. Batteries are considered to play an important role in this model. However, since they are costly, the total operational cost due to activating the power demands from the grid and batteries can be very high and hence the number of batteries need to be controlled [15]. The optimal control problem of the number batteries faced by the utility operator is addressed and a cost model is introduced.

\subsection{The Smart Grid System Without Batteries}

If the smart grid is considered without the use of batteries, it can be modeled as $M / M / \infty$. In this model and since the grid has a large capacity, it can be assumed that there are infinite number of servers available for service. Hence, all power demands will be activated upon arrival. Power demands are assumed to arrive with rate $\lambda$ and service rate is $\mu$. If the power requirement of each demand is 1 , the instantaneous power consumption, $X_{1}(t)$ is the number of active power demands at time $t$. Under these assumptions, $X_{1}$ is Markov jump process whose state space can be given by Figure 3.1 . 


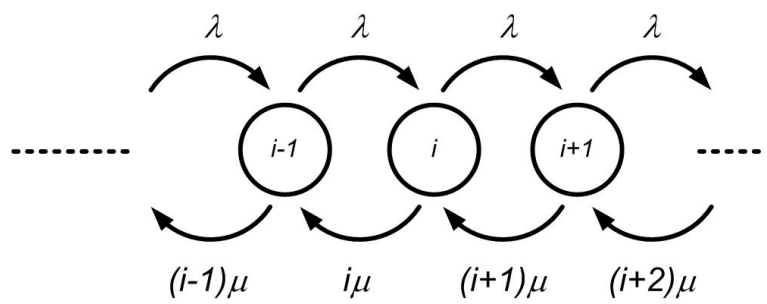

Figure 3.1: Continuous-time Markov chain of the smart grid without batteries

\subsection{The DES Model of the Smart Grid System with Batteries}

In the proposed DES model, power demands are assumed to arrive according to Poisson process with rate $\lambda$. They can get activated or served by any of the two energy sources, the main grid and batteries. The grid has infinite capacity. The total number of batteries that can be used simultaneously, can vary to accommodate the load of the current state. Empty batteries do not need to be recharged, instead they can be replaced by the time they get empty. The DES model is depicted in Figure 3.2.

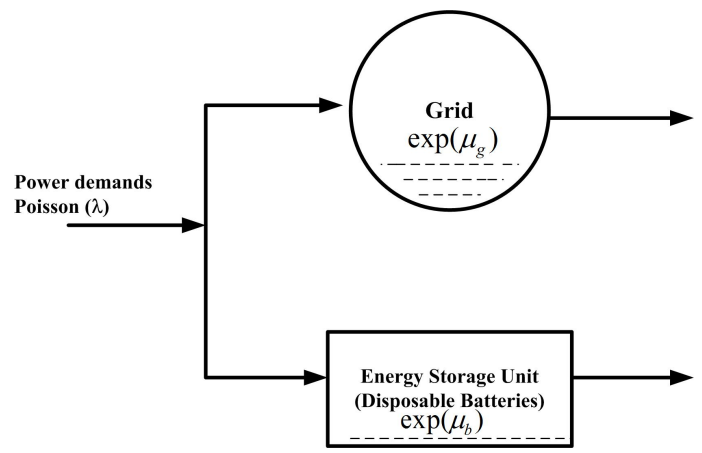

Figure 3.2: Dynamics of the proposed DES model

Service rates of the grid and batteries are $\mu_{g}>0$ and $\mu_{b}>0$ respectively. For $t \geq 0$, let $X_{1}(t)$ denote the total number of active power demands in the system at time $t$, which includes the demands that are served by batteries as well as the ones that are served by the grid. $X_{1}(t)$ then is the total instantaneous power consumption given that the power requirement of each task is 1 . Let $h_{1}(t)$ be the number of power 
demands served by batteries at time $t$. The function $h_{1}$ can in general depend on both $t$ and $X_{1}$ and is considered to be a control policy. Let $\Pi$ be the set of all possible policies. In addition, it is a must to have

$$
0 \leq h_{1}(t) \leq X_{1}(t)
$$

The dynamics of this system are captured by a continuous-time Markov chain with finite one-dimensional state space corresponding to the total number of power demands that are active in the system. The transition rates are given in Figure 3.3 . The departure rate is state-dependent, and is equal to $\left(X_{1}(t)-h_{1}(t)\right) \mu_{g}+h_{1}(t) \mu_{b}$.

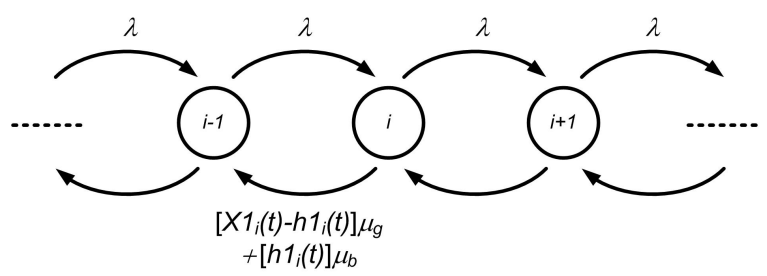

Figure 3.3: Continuous-time Markov chain of the proposed DES model

\subsection{Heavy Loads and Optimal Control Problem of the DES Model}

In this work a certain operational cost in the long-run is asymptotically minimized, for system in heavy load. For this, it is needed to assume that the arriving flow is large resulting in participating processes to be large as well. Consider a sequence of the smart grid systems with batteries parameterized by $n, n \rightarrow \infty$, (emphasized by using the superscript ${ }^{n}$ ) and assume that the arrival rate of the power demands is proportional to $n$, that is $\lambda^{n}=\lambda n$ for some $\lambda>0$. Define

$$
\bar{X}_{1}^{n}=\frac{1}{n} X_{1}^{n}, \quad \bar{h}_{1}^{n}=\frac{1}{n} h_{1}^{n} .
$$

Consider $\psi(\cdot)$ to be a continuously differentiable increasing convex function, and let $K \geq 0$ be some constant. For a certain policy $h_{1}^{n}$, and time $t$, define the ergodic 
operational cost as follows

$$
\mathcal{P}_{1}^{n, h_{1}^{n}}(t)=E\left(\frac{K}{t} \int_{0}^{t}\left(\bar{X}_{1}^{n}(s)-\bar{h}_{1}^{n}(s)\right) d s+\frac{1}{t} \int_{0}^{t} \psi\left(\bar{h}_{1}^{n}(s)\right) d s\right) .
$$

The first term represents the operational cost due to serving a number of power demands from the grid at time $t$, while the second term represents the operational cost due to serving a number of power demands by using disposable energy storage units. The interest is to find

$$
\mathcal{P}_{1}^{n, *}(t)=\inf _{h_{1}^{n} \in \Pi^{n}} \mathcal{P}_{1}^{n, h_{1}^{n}}(t)
$$

and the problem will be asymptotically solved under the assumption $n \rightarrow \infty$ and then $t \rightarrow \infty$.

\subsection{Fluid View of the DES Model}

Starting with the dynamics of the process $X_{1}^{n}$ and the corresponding fluid representation, the total number of power demands in the system at time $t, X_{1}^{n}$, must satisfy the relation

$$
X_{1}^{n}(t)=X_{1}^{n}(0)+A^{n}(t)-D_{g}^{n}(t)-D_{b}^{n}(t)
$$

where $X_{1}^{n}(0)$ is the initial number of power demands in the system, $A^{n}(t)$ is the total number of power demands that have arrived during the time interval $[0, t], D_{g}^{n}(t)$ is the total number of power demands that finish service by the grid during the same time interval, and $D_{b}^{n}(t)$ is the total number of power demands that finish service by batteries during the same time interval as well. Consider Poisson processes $S_{g}^{n}(t)$ and $S_{b}^{n}(t)$ with finite means $\mu_{g}>0$, and $\mu_{b}>0$, respectively. As discussed in Section 2, $X_{1}^{n}(t)$ can be equivalently represented as

$$
X_{1}^{n}(t)=X_{1}^{n}(0)+A^{n}(t)-S_{g}^{n}\left(\int_{s=0}^{t}\left(X_{1}^{n}(s)-h_{1}^{n}(s)\right) d s\right)-S_{b}^{n}\left(\int_{s=0}^{t} h_{1}^{n}(s) d s\right) .
$$

Using the notation $\bar{f}^{n}=f / n$ defined in Section 2.3.2, the relation 3.6 can be 
rewritten as

$$
\begin{aligned}
\bar{X}_{1}^{n}(t) & =\bar{X}_{1}^{n}(0)+\bar{A}^{n}(t)-\bar{S}_{g}^{n}\left(n \int_{s=0}^{t}\left(\bar{X}_{1}^{n}(s)-\bar{h}_{1}^{n}(s)\right) d s\right) \\
& -\bar{S}_{b}^{n}\left(n \int_{s=0}^{t} \bar{h}_{1}^{n}(s) d s\right)
\end{aligned}
$$

The heuristic derivation of the fluid model is based on the following lemma. For an arbitrary $T \geq 0$ and $\epsilon \in(0,1)$, define the event $B^{n}=B_{\epsilon, T}^{n}$ by

$$
B^{n}=B_{A}^{n} \cap B_{D_{g}}^{n} \cap B_{D_{b}}^{n}
$$

where

$$
\begin{gathered}
B_{A}^{n}=\left\{\sup _{t \in[0, T]}\left|\bar{A}^{n}(t)-\frac{\lambda^{n}}{n} t\right|<\epsilon\right\}, \\
B_{D_{g}}^{n}=\left\{\sup _{t \in[0, \kappa T]}\left|\frac{S_{g}(n t)}{n}-\mu_{g} t\right|<\epsilon\right\}, \\
B_{D_{b}}^{n}=\left\{\sup _{t \in[0, \kappa T]}\left|\frac{S_{b}(n t)}{n}-\mu_{b} t\right|<\epsilon\right\}
\end{gathered}
$$

and $\kappa=\kappa(T)=\lambda T+M+1$, where $M<\infty$ is such that $\bar{X}_{1}^{n}(0) \leq M$.

Lemma 1. Let $T \geq 0, \epsilon \in(0,1)$ be given. Then, on the event $B^{n}$, for every $n$,

$$
\left|\bar{D}_{g}^{n}(t)-\mu_{g} \int_{0}^{t}\left(\bar{X}_{1}^{n}(s)-\bar{h}_{1}^{n}(s)\right) d s\right| \vee\left|\bar{D}_{b}^{n}(t)-\mu_{b} \int_{0}^{t} \bar{h}_{1}^{n}(s) d s\right|<\epsilon,
$$

$t \in[0, T]$. Moreover, $P\left(B^{n}\right) \rightarrow 1$ as $n \rightarrow \infty$.

Proof: Proceeding as in Lemma 2.1 of $[2]$, the bound $\int_{0}^{T} \bar{X}_{1}^{n}(s) d s \leq \kappa T$ is guaranteed by the simple $X_{1}^{n}(t) \leq X_{1}^{n}(0)+A^{n}(t)$. The almost sure convergence (a.s convergence) $P\left(B_{A}^{n}\right) \rightarrow 1$ as $n \rightarrow \infty$ follows since $A^{n}$ is a Poisson process. The a.s convergence $P\left(B_{D_{g}}^{n}\right) \rightarrow 1$ as $n \rightarrow \infty$ holds since $S_{g}{ }^{n}$ is a Poisson process, see ( Chpt.5 of [11]) and (Theorem A.1 in Appendix A. For similar reasons and from Equation (3.1) the a.s convergence $P\left(B_{D_{b}}^{n}\right) \rightarrow 1$ as $n \rightarrow \infty$ follows as well. Consequently, $P\left(B^{n}\right) \rightarrow 1$ as $n \rightarrow \infty$ which this concludes the lemma. 
Introduce an error term $\bar{e}_{1}^{n}(t)$ as

$$
\begin{aligned}
\bar{e}_{1}^{n}(t) & =\left(\bar{A}^{n}(t)-\lambda t\right)+\left(\bar{D}_{g}^{n}(t)-\mu_{g} \int_{0}^{t}\left(\bar{X}_{1}^{n}(s)-\bar{h}_{1}^{n}(s)\right) d s\right) \\
& +\left(\bar{D}_{b}^{n}(t)-\mu_{b} \int_{0}^{t} \bar{h}_{1}^{n}(s) d s\right)
\end{aligned}
$$

and rewrite (3.7) as

$$
\bar{X}_{1}^{n}(t)=\bar{X}_{1}^{n}(0)+\lambda t-\mu_{g} \int_{s=0}^{t}\left(\bar{X}_{1}^{n}(s)-\bar{h}_{1}^{n}(s)\right) d s-\mu_{b} \int_{s=0}^{t} \bar{h}_{1}^{n}(s) d s+\bar{e}_{1}^{n}(t)
$$

implying that, at least on the event $B^{n}$, for $\epsilon$ small enough, the scaled dynamics is almost deterministic. 


\section{Chapter 4}

\section{Solving The Optimal Control Problem of the DES Model}

In this chapter a fluid approximation of the DES-model is presented and an asymptotically equivalent deterministic control problem is introduced. The latter is shown to have an easy solution: by using Jensen's Inequality the lower bound of the total operational cost is first identified, and then it is shown that the lower bound can be attained by a certain simple scheduling policy.

\subsection{The Deterministic Optimal Control Problem of the DES Model}

Section 3.4, and Equation (3.11) suggests deterministic fluid dynamics of $\bar{X}_{1}^{n}$ in the case when the error term is close to zero. In this section a deterministic version of (3.11), (3.3)-(3.4) is considered first and then the asymptotical equivalence between both problems is showed. Assume that the controlled process $X_{1}$ behaves like

$$
X_{1}(t)=X_{1}(0)+\lambda t-\mu_{g} \int_{s=0}^{t}\left(X_{1}(s)-h_{1}(s)\right) d s-\mu_{b} \int_{s=0}^{t} h_{1}(s) d s
$$

For a given control policy $h_{1}$, introduce the cost

$$
\mathcal{P}_{1}^{h_{1}}(t)=\frac{K}{t} \int_{0}^{t}\left(X_{1}(s)-h_{1}(s)\right) d s+\frac{1}{t} \int_{0}^{t} \psi\left(h_{1}(s)\right) d s
$$


The interest is to find

$$
\mathcal{P}_{1}^{*}=\inf _{h_{1} \in \Pi} \lim _{t \rightarrow \infty} \mathcal{P}_{1}^{h_{1}}(t) .
$$

In the next two sections the deterministic optimal control problem is completely solved.

\subsubsection{Part 1 of Solution. The Lower Bound on the Cost}

For a convex function $\psi$ an easy application of Jensen's Inequality (Theorem A.2 in Appendix A, suggests

$$
\frac{1}{t} \int_{0}^{t} \psi\left(h_{1}(s)\right) d s \geq \psi\left(\frac{1}{t} \int_{0}^{t} h_{1}(s) d s\right)
$$

As a result, the cost 4.2 can be bounded from below by

$$
P_{1}^{h_{1}}(t) \geq \frac{K}{t} \int_{0}^{t} X_{1}(s) d s-\frac{K}{t} \int_{0}^{t} h_{1}(s) d s+\psi\left(\frac{1}{t} \int_{0}^{t} h_{1}(s) d s\right)
$$

To find the final form of the lower bound on the cost, the following lemma is needed. For $X_{1}$ and $h_{1}$ are defined in Section 3.2 where $X_{1}$ and $h_{1}$ satisfy the Inequality (3.1). Also, recall that $M<\infty$ is such that $\bar{X}_{1}^{n}(0) \leq M$.

Lemma 2. For $t \geq 0$, there exists a constant $c_{1}>0$ such that total number of power demands in the system, $X_{1}(t)$ is bounded by

$$
X_{1}(t) \leq c_{1}
$$

and in particular, $\frac{X_{1}(t)}{t} \rightarrow 0$ as $t \rightarrow \infty$.

Proof: The standard comparison principle for the ODE (Theorem A.3 in Appendix A can be applied to Equation (4.1) as follows: Let $X_{1}(t)$ and $Y(t)$ be respectively solutions of

$$
\frac{d X_{1}(t)}{d t}=F_{1}\left(t, X_{1}(t)\right)=\lambda-\mu_{g} X_{1}(t)+\left(\mu_{g}-\mu_{b}\right) h_{1}(t)
$$


and

$$
\frac{d Y(t)}{d t}=F_{2}(t, Y(t))=\lambda-\mu Y(t)
$$

Observe that the functions $F_{1}$ and $F_{2}$ satisfy $F_{1}\left(t, X_{1}\right) \leq F_{2}\left(t, X_{1}\right)$. Indeed, if $\mu_{b} \leq \mu_{g}$ then $F_{1}\left(t, X_{1}\right) \leq F_{2}\left(t, X_{1}\right)=\lambda-\mu_{g} X_{1}+\left(\mu_{g}-\mu_{b}\right) X_{1}$ because $\left(\mu_{g}-\mu_{b}\right) h_{1}(t) \leq$ $\left(\mu_{g}-\mu_{b}\right) X_{1}(t)$ since $h_{1} \leq X_{1}$ by definition. On the other hand, if $\mu_{g} \leq \mu_{b}$ then $F_{1}\left(t, X_{1}\right) \leq F_{2}\left(t, X_{1}\right)$ since $\left(\mu_{g}-\mu_{b}\right) h_{1}(t) \leq 0$ is always true. As a result, assuming $X_{1}(0)=Y(0)$, it is a must to have $X_{1}(t) \leq Y(t)$ for all $t \geq 0$. In turn, the differential equation for $Y$ can be explicitly solved as

$$
Y(t)=\frac{\lambda}{\mu}-\left(\frac{\lambda}{\mu}-Y(0)\right) e^{-\mu t}
$$

obviously $Y(t) \leq c_{1}$ for a constant $c_{1}>0$. All the above imply that $X_{1}(t) \leq c_{1}$ for all $t \geq 0$.

Inequality 4.5 can be restated as a function of a control variable that can be used to minimize the total operational cost. From Equation 4.1,

$$
\frac{1}{t} \int_{0}^{t} X_{1}(s) d s=\frac{1}{t \mu_{g}}\left(X_{1}(0)-X_{1}(t)+\lambda t\right)+\left(\frac{\mu_{g}-\mu_{b}}{\mu_{g}}\right) \frac{1}{t} \int_{0}^{t} h_{1}(s) d s
$$

and

$$
\frac{1}{t} \int_{0}^{t} X_{1}(s) d s=\eta(t)+\left(\frac{\mu_{g}-\mu_{b}}{\mu_{g}}\right) H_{1}(t)
$$

Where, $\eta(t)$ and $H_{1}(t)$ can be set as

$$
\eta(t)=\frac{1}{t \mu_{g}}\left(X_{1}(0)-X_{1}(t)+\lambda t\right), \quad H_{1}(t)=\frac{1}{t} \int_{0}^{t} h_{1}(s) d s .
$$

In the limit, $\eta(t) \rightarrow \lambda / \mu_{g}$ as $t \rightarrow \infty$, because $X_{1}(0) / t \rightarrow 0$ as $t \rightarrow \infty$ and $X_{1}(t) / t \rightarrow 0$ as $t \rightarrow \infty$ since $X_{1}$ is bounded by a constant $=\lambda / \mu$. Finally,

$$
P_{1}^{h_{1}}(t) \geqslant L^{h_{1}}(t):=K \eta(t)-K \frac{\mu_{b}}{\mu_{g}} H_{1}(t)+\psi\left(H_{1}(t)\right) .
$$

From Equation 4.13), as $t \rightarrow \infty$, the lower bound takes the form 


$$
L\left(\bar{H}_{1}\right):=K \frac{\lambda}{\mu_{g}}-K \frac{\mu_{b}}{\mu_{g}} \bar{H}_{1}+\psi\left(\bar{H}_{1}\right), \quad \bar{H}_{1}=\lim _{t \rightarrow \infty}\left(\frac{1}{t} \int_{0}^{t} h_{1}(s) d s\right)
$$

\subsubsection{Part 2 of Solution. The Optimal Policy for the DES Model}

To continue with the solution, the optimal policy is obtained as follows. Let $h_{1}^{*}$ be a minimizer of Equation (4.14) over $\bar{H}_{1}$. Consider the policy that keeps constant $h_{1}(t) \equiv h_{1}^{*}$. To check that the lower bound is achievable by $h_{1}^{*}$, substitute $h_{1}^{*}$ in Equation 4.2, and take the limit as $t \rightarrow \infty$, giving

$$
\begin{aligned}
& \lim _{t \rightarrow \infty}\left(\frac{K}{t} \int_{0}^{t}\left(X_{1}(s)-h_{1}^{*}\right) d s\right)+\lim _{t \rightarrow \infty}\left(\frac{1}{t} \int_{0}^{t} \psi\left(h_{1}^{*}\right) d s\right) \\
& =K \lim _{t \rightarrow \infty}\left(\frac{1}{t} \int_{0}^{t} X_{1}(s) d s\right)-K h_{1}^{*}+\psi\left(h_{1}^{*}\right) \\
& =K \frac{\lambda}{\mu_{g}}-K \frac{\mu_{b}}{\mu_{g}} h_{1}^{*}+\psi\left(h_{1}^{*}\right)=: \rho^{*} .
\end{aligned}
$$

Thus

$$
\lim _{t \rightarrow \infty} P^{h_{1}^{*}}(t)=\rho^{*}
$$

Finally, the nonnegativity of Equation 4.14 of the lower bound can be proved as follows. By definition, one have $0 \leq h_{1}(t) \leq X_{1}(t)$, then it is true to have

$$
0 \leq \frac{1}{t} \int_{0}^{t} h_{1}(s) d s \leq \frac{1}{t} \int_{0}^{t} X_{1}(s) d s
$$

In addition, from 4.11

$$
\frac{1}{t} \int_{0}^{t} X_{1}(s) d s \rightarrow\left(\frac{\lambda}{\mu_{g}}+\frac{\mu_{g}-\mu_{b}}{\mu_{g}} \bar{H}_{1}\right) \text { as } t \rightarrow \infty .
$$


Combining with 4.17), one can have

$$
\bar{H}_{1} \leq\left(\frac{\lambda}{\mu_{g}}+\frac{\mu_{g}-\mu_{b}}{\mu_{g}} \bar{H}_{1}\right)
$$

which implies

$$
\frac{\mu_{b}}{\mu_{g}} \bar{H}_{1} \leq \frac{\lambda}{\mu_{g}}
$$

Then $\bar{H}_{1} \leq \lambda / \mu_{b}$ which leads to the required result, since for $K>0$

$$
K \frac{\lambda}{\mu_{g}}-K \frac{\mu_{b}}{\mu_{g}} \bar{H}_{1} \geqslant 0
$$

and,

$$
\min _{\bar{H}_{1} \in R}\left(K \frac{\lambda}{\mu_{g}}-K \frac{\mu_{b}}{\mu_{g}} \bar{H}_{1}+\psi\left(\bar{H}_{1}\right)\right) \geq 0 \quad \text { for } \quad \bar{H}_{1} \in\left[0 ; \frac{\lambda}{\mu_{b}}\right]
$$

The full solution of the deterministic optimal control problem leads to stating the following theorem which summarizes the main result of this work.

Theorem 4.1. Let $h_{1}^{*}$ be a minimizer of the function $L(u):=K \frac{\lambda}{\mu_{g}}-K \frac{\mu_{b}}{\mu_{g}} u+\psi(u)$ in the interval $\left[0 ; \frac{\lambda}{\mu_{b}}\right]$. Then, an optimal policy for the deterministic control problem is to have $h^{\star}(t)=\min \left\{X_{1}(t), h_{1}^{*}\right\}$, and the optimal cost is given by $\rho^{*}=\lim _{t \rightarrow \infty} P^{h_{1}^{*}}(t)$ from 4.16.

Moreover, if $X_{1}(0) \geq h_{1}^{*}$, then $h_{1}^{\star} \equiv h_{1}^{*}$, otherwise there exists a finite $\delta>0$ such that $h_{1}^{\star}(t)=X_{1}(t)$ for $[0, \delta]$ and $h_{1}^{\star}(t)=h_{1}^{*}$ for $t \geq \delta$.

\subsection{Stochastic Model. Interpretation of the Policy}

The above analysis suggests that in the original problem the number of batteries $h_{1}^{n}(t)$ at any time $t$ should be taken close to $n h_{1}^{*}$, with $h_{1}^{*}$ from Theorem 4.1. Indeed, let $h_{1}^{n, *}(t)=\min \left\{X_{1}^{n}(t), n h_{1}^{*}\right\}$. The following conjecture is believed to follow from Theorem 5.1 in [2], yet the proof is omitted. 


\section{Conjecture}

$$
\liminf _{t \rightarrow \infty} \liminf _{n \rightarrow \infty} \mathcal{P}_{1}^{n, *}(t)=\lim _{t \rightarrow \infty} \lim _{n \rightarrow \infty} \mathcal{P}_{1}^{n, h_{1}^{n, *}}(t)=\rho^{*}
$$

for (recall 4.16) $\rho^{*}:=K \frac{\lambda}{\mu_{g}}-K \frac{\mu_{b}}{\mu_{g}} h_{1}^{*}+\psi\left(h_{1}^{*}\right)$.

The proposed optimal policy can be achieved through two steps (for clarity, the superscript $n$ is omitted). First, identify the minimizer $h_{1}^{*}$. Second, since the policy $h_{1}^{*}$ turned out to be constant perform the following: power demands can be activated by batteries first, where the maximum number of power demands that can be activated simultaneously by batteries at any time $t, h_{1}(t)$ is controlled by allowing only a max of $h_{1}^{*}$ units to be used. Empty batteries get constantly replaced by new ones. The rest of the power demands can be activated by the grid. The dynamics of the DES model given the control policy $h_{1}^{*}$ is depicted in Figure 4.1 .

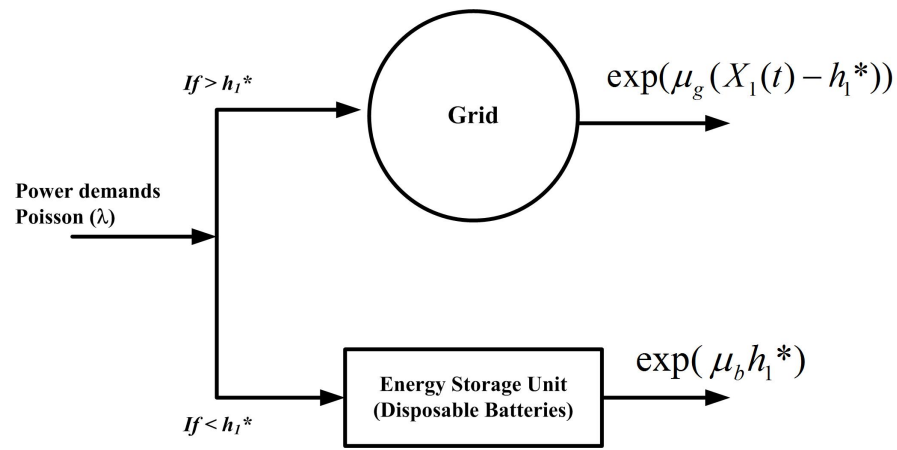

Figure 4.1: Dynamics of the proposed DES model given the control policy $h_{1} *$

The limiting probabilities of the DES model given the control policy $h_{1}^{*}$ can be obtained as follows: Let $\left(P_{0}, P_{1}, \ldots, P_{i}, \ldots\right)$ denote the limiting probabilities, where $P_{i}$ is the long run limiting probability that the system has $i$ power demands. The departure rate is state-dependent, and is equal to $\left(X_{1}(t)-h_{1}^{*}\right) \mu_{g}+h_{1}^{*} \mu_{b}$. The transition rates are given in Figure 4.2 . 


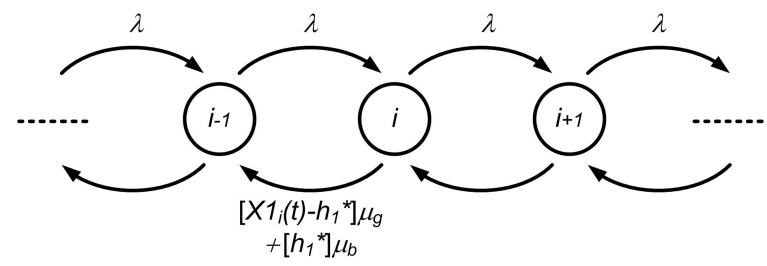

Figure 4.2: Continuous-time Markov chain of the proposed DES model given the control policy $h_{1} *$

From the balance equation, at state $i$ :

$$
P_{i}=\frac{\lambda P_{i-1}+\left(\left(X_{1_{i+1}}-h_{1}^{*}\right) \mu_{g}+h_{1}^{*} \mu_{b}\right) P_{i+1}}{\left(\lambda+\left(X_{1_{i}}-h_{1}^{*}\right) \mu_{g}+h_{1}^{*} \mu_{b}\right)}
$$

The average total number of power demands in the system can be computed as

$$
E\left(X_{1}\right)=\sum_{i} i P_{i}
$$




\section{Chapter 5}

\section{Generalizations of the Smart Grid System}

In this chapter, two generalizations of the DES model analyzed in previous chapters are considered. The first one (referred to as N-DES model) will demonstrate that almost all of the previous results remain true if a homogeneous Poisson arrival process is replaced by a non-homogeneous one. The second model (referred to as the RES

model), is more involved and introduces rechargeable batteries. The model suggests that the fully charged batteries can be used at any time and then can be recharged by the grid.

\subsection{The N-DES Model of the Smart Grid System}

In the N-DES model the assumption of stationary increments of an arrival process is relaxed, and the power demands are assumed to arrive according to a non-homogeneous Poisson process with time-dependent rate. In general, the nonhomogeneous Poisson process is important counting process for modelling purposes. It allows for the possibility that the arrival rate to vary with time [16].

The heavy load assumption is formulated as having the rate $\lambda^{n}(t)=n \lambda(t)$ for some deterministic function $\lambda(t)$. The stochastic problem is the same as in (3.3)(3.4), hence it makes sense to discuss the deterministic fluid setting only. Namely, the principal change will be to replace the scaled arrival process $\bar{A}^{n}$ by a process $\Lambda$, 
satisfying $\Lambda(t)=\int_{0}^{t} \lambda(s) d s$. In addition, the following assumption is imposed

$$
\lim _{t \rightarrow \infty}\left(\frac{1}{t} \int_{0}^{t} \lambda(s) d s\right)=\bar{\lambda}<\infty
$$

The fluid dynamics will look like as

$$
X_{1}(t)=X_{1}(0)+\Lambda(t)-\mu_{g} \int_{0}^{t}\left(X_{1}(s)-h_{1}(s)\right) d s-\mu_{b} \int_{0}^{t} h_{1}(s) d s .
$$

Once again, by Jensen's Inequality, the total operational cost is bounded by

$$
\mathcal{P}_{1}^{h_{1}}(t) \geq \frac{K}{t} \int_{0}^{t} X_{1}(s) d s-\frac{K}{t} \int_{0}^{t} h_{1}(s) d s+\psi\left(\frac{1}{t} \int_{0}^{t} h_{1}(s) d s\right) .
$$

In addition, from Equation (5.2), the total number of power demands can be stated as a function of the control variable $H_{1}(t)$. Let

$$
H_{1}(t)=\frac{1}{t} \int_{0}^{t} h_{1}(s) d s, \quad \eta(t)=\frac{1}{t \mu_{g}}\left(X_{1}(0)-X_{1}(t)+\int_{0}^{t} \lambda(s) d s\right)
$$

then,

$$
\frac{1}{t} \int_{0}^{t} X_{1}(s) d s=\eta(t)+\frac{\mu_{g}-\mu_{b}}{\mu_{g}} H_{1}(t) .
$$

The cost can be bounded by

$$
\mathcal{P}_{1}^{h_{1}}(t) \geq L^{h_{1}}(t)=K \eta(t)-K \frac{\mu_{b}}{\mu_{g}} H_{1}(t)+\psi\left(H_{1}(t)\right)
$$

From (5.1) one can have

$$
\lim _{t \rightarrow \infty} \eta(t)=\lim _{t \rightarrow \infty} \frac{X_{1}(0)-X_{1}(t)}{\mu_{g} t}+\lim _{t \rightarrow \infty} \frac{\int_{0}^{t} \lambda(s) d s}{\mu_{g} t}=\bar{\lambda} / \mu_{g}
$$

The lower bound takes the form

$$
L\left(\bar{H}_{1}\right):=K \frac{\bar{\lambda}}{\mu_{g}}-K \frac{\mu_{b}}{\mu_{g}} \bar{H}_{1}+\psi\left(\bar{H}_{1}\right)
$$

and, the optimal policy is the one that keeps constant $h_{1}(t) \equiv h_{1}^{*}$, where $h_{1}^{*}$ be a 
minimizer of Equation (5.8) over $\bar{H}$.

\subsection{The RES Model of the Smart Grid System}

In the RES model rechargeable batteries are used instead of the disposable ones, and an additional component is added to the cost. For $t \geq 0$, let $X_{2}(t)$ denote the total number of active power demands in the system at time $t$, which includes the demands that are served by batteries as well as the power demands that are served by the grid. Also, let $h_{2}(t)$ be the number of power demands served by batteries at time $t$. In addition, let $B(t)$, be the capacity of batteries (the total number of full batteries) at time $t$. Empty batteries can be recharged from the grid with rate $\alpha>0$. The RES model is depicted in Figure 5.1 .

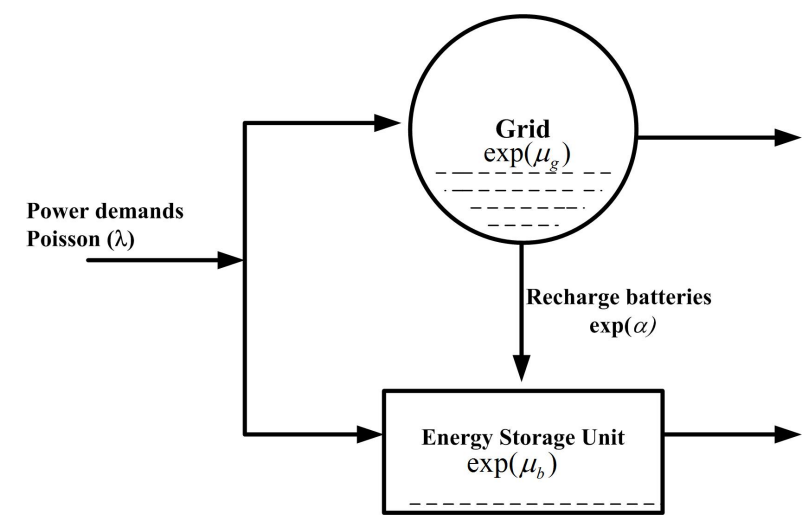

Figure 5.1: Dynamics of the proposed RES model

The RES model is captured by a continuous-time Markov chain with twodimensional state space: one dimension corresponding to the total number of power demands that are active in the system and another dimension corresponding to the total number of full batteries. The transition rates are given in Figure 5.2 . 


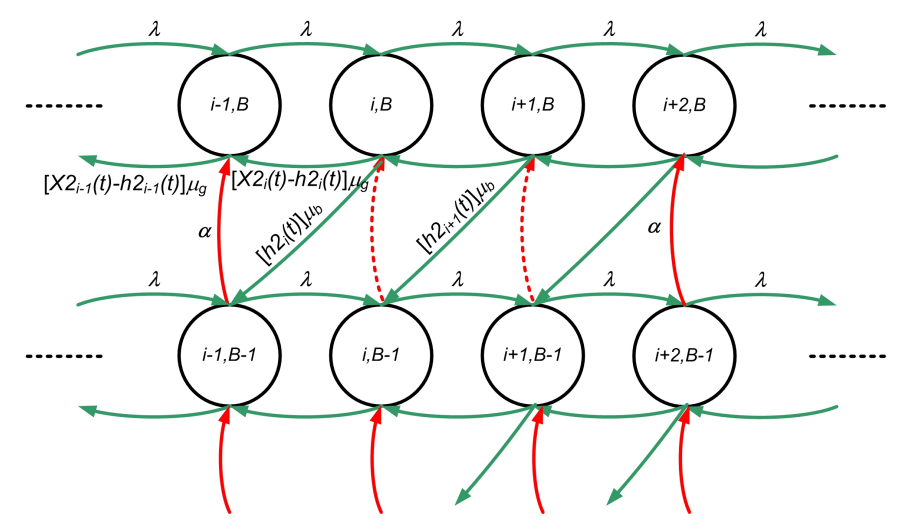

Figure 5.2: Continuous-time Markov chain of the proposed RES model

\subsection{Heavy Loads and Optimal Control Problem of the RES Model}

The operational cost of the RES model in the long-run is asymptotically minimized, for system in heavy load as well. Consider a sequence of the smart grid systems with rechargeable batteries parameterized by $n, n \rightarrow \infty$. Let $C(\cdot)$ be a continuously differentiable increasing convex function, and let $K_{1} \geq 0, K_{2} \geq 0$ be some constants. For a given a control policy $h_{2}=\left\{h_{2}(s), s \geq 0\right\}$, define the ergodic operational cost as follows

$\mathcal{P}_{2}^{n, h_{2}}(t)=E\left(\frac{K_{1}}{t} \int_{0}^{t}\left(\bar{X}_{2}^{n}(s)-\bar{h}_{2}^{n}(s)\right) d s+\frac{K_{2}}{t} \int_{0}^{t}\left(\bar{B}(0)^{n}(s)-\bar{B}^{n}(s)\right) d s+\frac{1}{t} \int_{0}^{t} C\left(\bar{h}_{2}^{n}(s)\right) d s\right)$

Where the operational cost in this case incorporates linear costs on the number of power demands served by the grid, the number of empty batteries that got recharged by the grid, and the convex cost on number of power demands served by batteries. The interest is to find

$$
\mathcal{P}_{2}^{n, *}(t)=\inf _{h_{2}^{n} \in \Pi^{n}} \mathcal{P}_{2}^{n, h_{2}}(t)
$$

and the problem will be asymptotically solved under the assumption $n \rightarrow \infty$ and then $t \rightarrow \infty$. 


\subsection{Fluid View of the RES Model}

This section defines (compare with Section 3.4) two equations that describe the dynamics of the RES model, since both active power demands and the number of full batteries have to be captured.

The process, $X_{2}^{n}$, must satisfy the following relation

$$
X_{2}^{n}(t)=X_{2}^{n}(0)+A^{n}(t)-D_{g}^{n}(t)-D_{b}^{n}(t)
$$

where $X_{2}^{n}(0)$ is the initial number of power demands in the system, $A^{n}(t)$ is the total number of power demands that have arrived during the time interval $[0, t], D_{g}^{n}(t)$ is the total number of power demands that can potentially finish service by the grid during the same time interval, and $D_{b}^{n}(t)$ is the total number of power demands that can potentially finish service by batteries in the same time interval as well. Consider Poisson processes $S_{g}^{n}(t)$ and $S_{b}^{n}(t)$ with finite means $\mu_{g}, \mu_{g}>0$, and $\mu_{b}, \mu_{b}>0$, respectively. $X_{2}^{n}(t)$ can be also given by

$$
\begin{aligned}
X_{2}^{n}(t) & =X_{2}^{n}(0)+A(t)-S_{g}^{n}\left(\int_{s=0}^{t}\left(X_{2}^{n}(s)-h_{2}^{n}(s)\right) d s\right) \\
& -S_{b}^{n}\left(\int_{s=0}^{t} h_{2}^{n}(s) d s\right) .
\end{aligned}
$$

Similarly, $X_{2}^{n}$ and $h_{2}^{n}$ must satisfy the Equation (3.1) of $X_{1}^{n}$ and $h_{1}^{n}$ respectively.

The process, $B^{n}(t)$, must satisfy the following relation

$$
B^{n}(t)=B^{n}(0)-D_{b}^{n}(t)+A_{g}^{n}(t)
$$

where $B^{n}(0)>0$ is the initial capacity of batteries, and $B^{n}(t)$ must satisfy $0 \leq$ $B^{n}(t) \leq B^{n}(0) . D_{b}^{n}(t)$ is the total number of power demands that get serviced by the batteries with rate $\mu_{b}$ during the time interval $[0, t]$, and $A_{g}^{n}(t)$ is the total number of batteries that get recharged by the grid with rate $\alpha$ during the same time interval. Consider Poisson process $F^{n}(t)$ with finite mean $\alpha, \alpha>0$. The capacity of batteries at time $t, B^{n}(t)$ can be represented by 


$$
B^{n}(t)=B^{n}(0)-S_{b} \int_{0}^{t} h_{2}^{n}(s) d s+F^{n}\left(\int_{0}^{t}\left(B^{n}(0)-B^{n}(s)\right) d s\right) .
$$

Applying scaling to Equations (5.12) and (5.14), one can get

$$
\begin{aligned}
\bar{X}_{2}^{n}(t) & =\bar{X}_{2}^{n}(0)+\bar{A}^{n}(t)-\bar{S}_{g}^{n}\left(n \int_{s=0}^{t}\left(\bar{X}_{2}^{n}(s)-\bar{h}_{2}^{n}(s)\right) d s\right) \\
& -\bar{S}_{b}^{n}\left(n \int_{s=0}^{t} \bar{h}_{2}^{n}(s) d s\right)
\end{aligned}
$$

and,

$$
\bar{B}^{n}(t)=\bar{B}^{n}(0)-\bar{S}_{b}^{n} \int_{0}^{t} \bar{h}_{2}^{n}(s) d s+\bar{F}^{n}\left(n \int_{0}^{t}\left(\bar{B}^{n}(0)-\bar{B}^{n}(s)\right) d s\right) .
$$

From Lemma (1) presented in Section (3.4), the convergence of the scaled Poisson processes $\bar{A}^{n}, \bar{F}^{n}, \bar{S}_{g}^{n}$, and $\bar{S}_{b}^{n}$ can be also proved. Introduce the following error terms

$$
\begin{aligned}
\bar{e}_{2}^{n}(t) & =\left(\bar{A}^{n}(t)-\bar{\lambda}^{n} t\right)+\left(\bar{D}_{g}^{n}(t)-\mu_{g} \int_{0}^{t}\left(\bar{X}_{2}^{n}(s)-\bar{h}_{2}^{n}(s)\right) d s\right) \\
& +\left(\bar{D}_{b}^{n}(t)-\mu_{b} \int_{0}^{t} \bar{h}_{2}^{n}(s) d s\right)
\end{aligned}
$$

and,

$$
\bar{e}_{3}^{n}(t)=\left(\bar{D}_{b}^{n}(t)-\mu_{b} \int_{0}^{t} \bar{h}_{2}^{n}(s) d s\right)+\left(\bar{A}_{g}^{n}(t)-\alpha \int_{0}^{t}\left(\bar{B}^{n}(0)-\bar{B}^{n}(s)\right) d s\right)
$$

The processes $\bar{X}_{2}^{n}$ and $\bar{B}^{n}$ in the limit can respectively given by :

$$
\begin{aligned}
\bar{X}_{2}^{n}(t) & =\bar{X}_{2}^{n}(0)+\bar{\lambda}^{n} t-\mu_{g} \int_{s=0}^{t}\left(\bar{X}_{2}^{n}(s)-\bar{h}_{2}^{n}(s)\right) d s \\
& -\mu_{b} \int_{s=0}^{t} \bar{h}_{2}^{n}(s) d s+\bar{e}_{2}^{n}(t),
\end{aligned}
$$

and, 


$$
\bar{B}^{n}(t)=\bar{B}^{n}(0)-\mu_{b} \int_{0}^{t} \bar{h}_{2}^{n}(s) d s+\alpha \int_{0}^{t}\left(\bar{B}^{n}(0)-\bar{B}^{n}(s)\right) d s+\bar{e}_{3}^{n}(t)
$$

\subsubsection{RES Model. Fluid Optimization}

In this section, the fluid dynamics (that is, the relations involving the scaled limiting quantities $\left.\bar{f}^{n}=f / n\right)$, and the fluid optimization problem are stated. Given that the error terms $\bar{e}_{2}^{n}(t)$ and $\bar{e}_{2}^{n}(t)$ can be neglected, one can have

$$
\begin{gathered}
X_{2}(t)=X_{2}(0)+\lambda t-\mu_{g} \int_{0}^{t}\left(X_{2}(s)-h(s)\right) d s-\mu_{b} \int_{0}^{t} h(s) d s, \\
B(t)=B(0)-\mu_{b} \int_{0}^{t} h(s) d s+\alpha \int_{0}^{t}(B(0)-B(s)) d s .
\end{gathered}
$$

The deterministic ergodic total operational cost can be given by

$$
\begin{aligned}
P_{2}^{h_{2}}(t) & =\frac{K_{1}}{t} \int_{0}^{t}\left(X_{2}(s)-h(s)\right) d s+\frac{K_{2}}{t} \int_{0}^{t}(B(0)-B(s)) d s \\
& +\frac{1}{t} \int_{0}^{t} C\left(h_{2}(s)\right) d s
\end{aligned}
$$

As before, the interest is to find

$$
\mathcal{P}_{2}^{*}=\inf _{h_{2}} \lim _{t \rightarrow \infty} P_{2}^{h_{2}}(t)
$$

\subsubsection{The Optimal Policy of the RES Model}

Similar to Section 4.1.1 and by Jensen's Inequality, the total operational cost faced by the utility operator, $P_{2}^{h_{2}}(t)$, can be bounded from below by

$$
\begin{aligned}
P_{2}^{h_{2}}(t) \geq & \frac{K_{1}}{t} \int_{0}^{t} X_{2}(s) d s-\frac{K_{1}}{t} \int_{0}^{t} h_{2}(s) d s+\frac{K_{2}}{t} \int_{0}^{t}(B(0)-B(s)) d s \\
& +C\left(\frac{1}{t} \int_{0}^{t} h_{2}(s) d s\right) .
\end{aligned}
$$


The standard comparison theorem for ODE, (Theorem A.3 in Appendix A can also be applied to Equation (5.21) as in Section 4.1.1 to show that the total number of power demands at time $t, X_{2}$ is bounded by a constant $\lambda / \mu$. Inequality (5.25) can be restated as a function of a control variable that can be used to minimize the total operational cost as follows. From Equations (5.21) and (5.22), set

$$
H_{2}(t)=\frac{1}{t} \int_{0}^{t} h_{2}(s) d s, \quad \xi(t)=\frac{1}{t}(B(t)-B(0))
$$

and

$$
\mu_{b} H_{2}(t)+\xi(t)=\frac{\alpha}{t} \int_{0}^{t}(B(0)-B(s)) d s
$$

Where $\xi(t) \rightarrow 0$ as $t \rightarrow \infty$, because $B(\cdot)$ is bounded by definition. Equation (5.27) reflects that, in the long run, the average number of power demands that get served by batteries is equal to the average number of empty batteries that get recharged by the grid. Finally,

$$
P_{2}^{h_{2}}(t) \geq K_{1} \frac{1}{t} \int_{0}^{t} X_{2}(s) d s-K_{1} H_{2}(t)+K_{2} \frac{\mu_{b} H_{2}(t)+\xi(t)}{\alpha}+C\left(H_{2}(t)\right) .
$$

From (5.21)

$$
\frac{1}{t} \int_{0}^{t} X_{2}(s) d s=\eta(t)+\frac{\mu_{g}-\mu_{b}}{\mu_{g}} H_{2}(t)
$$

where

$$
\eta(t)=\frac{1}{t \mu_{g}}\left(X_{2}(0)-X_{2}(t)+\lambda t\right)
$$

Since that, in the limit, $\eta(t) \rightarrow \lambda / \mu_{g}$ as $t \rightarrow \infty$. The lower bound on the total operational cost, $L^{h_{2}}(t)$, can now be given as

$$
P_{2}^{h_{2}}(t) \geqslant L^{h_{2}}(t):=K_{1} \eta(t)-\frac{\mu_{b} K_{1}}{\mu_{g}} H_{2}(t)+K_{2} \frac{\mu_{b} H_{2}(t)+\xi(t)}{\alpha}+C\left(H_{2}(t)\right)
$$

and,

$$
L\left(\bar{H}_{2}\right):=K_{1} \frac{\lambda}{\mu_{g}}-K_{1} \frac{\mu_{b}}{\mu_{g}} \bar{H}_{2}+\frac{\mu_{b} K_{2}}{\alpha} \bar{H}_{2}+C\left(\bar{H}_{2}\right) .
$$


Let $h_{2}^{*}$ be a minimizer of $(5.32)$ over $\bar{H}_{2}$. Consider the policy that keeps constant $h_{2}(t) \equiv h_{2}^{*}$. To check that the lower bound is indeed achievable by $h_{2}^{*}$, substitute $H_{2}(t)$ by $h_{2}^{*}$ in Equation (5.24), giving

$$
\begin{aligned}
& \lim _{t \rightarrow \infty} P_{2}^{h_{2}^{*}}(t)=\lim _{t \rightarrow \infty}\left(\frac{K_{1}}{t} \int_{0}^{t}\left(X_{2}(s)-h_{2}^{*}\right) d s\right)+\lim _{t \rightarrow \infty}\left(\frac{K_{2}}{t} \int_{0}^{t}(B(0)-B(s)) d s\right) \\
&+\lim _{t \rightarrow \infty}\left(\frac{1}{t} \int_{0}^{t} C\left(h_{2}^{*}\right) d s\right) . \\
&=K_{1} \lim _{t \rightarrow \infty}\left(\frac{1}{t} \int_{0}^{t}\left(X_{2}(s)\right) d s\right)-K_{1} h_{2}^{*}+\left(\frac{\mu_{b} K_{2}}{\alpha} h_{2}^{*}\right)+\lim _{t \rightarrow \infty}\left(\frac{K_{2}}{\alpha t}(B(t)-B(0))\right)+C\left(h_{2}^{*}\right) .
\end{aligned}
$$

$$
=K_{1} \frac{\lambda}{\mu_{g}}-K_{1} \frac{\mu_{b}}{\mu_{g}} h_{2}^{*}+\frac{\mu_{b} K_{2}}{\alpha} h_{2}^{*}+C\left(h_{2}^{*}\right)=\rho^{*} .
$$

$L^{h_{2}}(t)$ is the lower bound on the cost. Let $t \rightarrow \infty$ and take the limit. To check that Equation 5.32 is indeed positive, the following proof is given. From Equations 4.17 . 4.22 , it can be shown that

$$
K_{1} \frac{\lambda}{\mu_{g}}-K_{1} \frac{\mu_{b}}{\mu_{g}} \bar{H}_{2} \geqslant 0
$$

and

$$
\min _{\bar{H}_{2} \in R}\left\{K_{1} \frac{\lambda}{\mu_{g}}-K_{1} \frac{\mu_{b}}{\mu_{g}} \bar{H}_{2}+\frac{\mu_{b} K_{2}}{\alpha} \bar{H}_{2}+C\left(\bar{H}_{2}\right)\right\}>0,
$$

where, $\bar{H}_{2} \in\left[0 ; \frac{\lambda}{\mu_{b}}\right]$ which concludes the full solution of the deterministic optimal control problem of the RES model. 


\section{Chapter 6}

\section{Simulation Results}

In this chapter several simulations are carried out to verify that the method of fluid approximation is effective for estimating the behaviour for different queueing models under the heavy load conditions. (See Appendix B). Examples are given for the $M / M / N$ queuing model in addition to the proposed DES, N-DES, and RES models of the smart grid system with batteries. The numerical results for the behaviour of their fluid models are compared to simulation results of their respective scaled stochastic models. In general, it is shown that although the deterministic fluid model does not capture important stochastic fluctuations, it provides remarkably accurate approximations for the key processes of the many-servers queuing model and the models of the smart grid. Additionally, the simulations of each model of the smart grid system were run to compere between the stochastic performance of each model under the proposed policy and the deterministic performance. The optimal total operational cost plots of each model are obtained and shown to reach the lower bound in the limit.

\subsection{Simulation Results of $M / M / N$ Queuing Model}

A simulation experiment is run for $M / M / N$ queuing model described in Section 2.3 with the goal of comparing the fluid behaviour of the simulated stochastic total number of customers in the system $Q^{n}(t)$ of Equation 2.5 and the fluid $\bar{Q}(t)$ of Equation (2.6). In this experiment, customers arrive with very big rate $\lambda n=0.8 n$, the 
number of servers is $N n \simeq n$, and the service rate is $\mu=1$. The initial number of customers in the system, $Q(0)$, is assumed to be zero. Figure 6.1 shows the comparison for $n=100$ and $n=1000$. It can be observed that the larger the system, the better the fluid approximates the key performance measures such as $Q$. Figure 6.1 clearly shows that $n=1000$ is large enough in the sense that the simulated $Q$ is very close to the fluid approximation. However, the fluid approximation is some how rough when $n=100$.

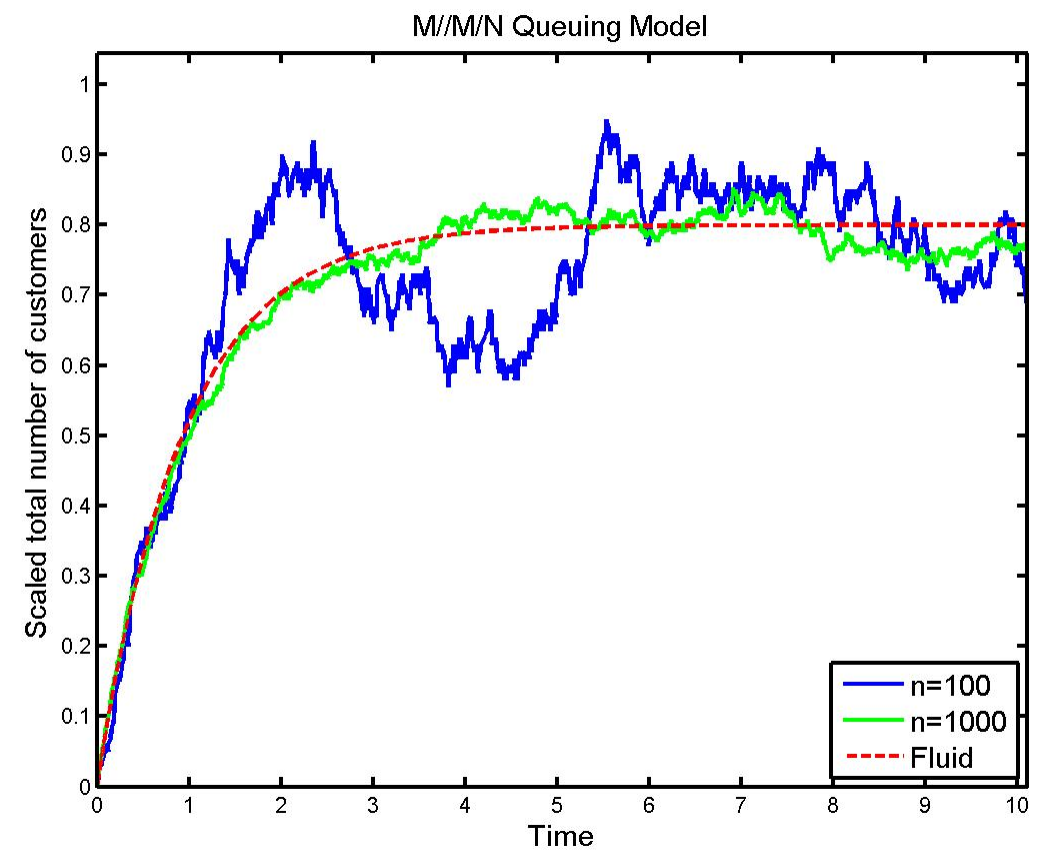

Figure 6.1: A comparison of one sample path of $\bar{Q}^{n}(t)$ to fluid $Q(t)$ of the $M / M / N$ queuing model

\subsection{Simulation Results of the DES Model}

To validate the fluid approximation of the DES model described in Section 3.2, a simulation experiment is run as follows. In this experiment, the grid capacity is suppose to be infinity, but it is represented by $N=2200$. The arrival rate $\lambda n=2 n$ power demands/hour is set to be very big as well where $n$ takes values 100, and 1000. The initial number of power demands in the system, $X_{1}(0)$, is assumed to 
be zero, the constant $K_{1}=1$, the average service rates from grid and batteries are $\mu_{g}=2$ power demands/hour, and $\mu_{b}=0.1$ power demands/hour respectively. Let the average power requirement per demand be $1 \mathrm{~kW}$, and $\psi\left(h_{1}(s)\right)=h_{1}^{2}(s)$. From Equation 4.14,

$$
L\left(\bar{H}_{1}\right):=K_{1} \frac{\lambda}{\mu_{g}}-K_{1} \frac{\mu_{b}}{\mu_{g}} \bar{H}_{1}+\left(\bar{H}_{1}^{2}\right)
$$

and

$$
\frac{d L\left(\bar{H}_{1}\right)}{d \bar{H}_{1}}=-K_{1} \frac{\mu_{b}}{\mu_{g}}+2 \bar{H}_{1} .
$$

The optimal capacity of batteries is set to equal to $h_{1}^{*} n$ and $h_{1}^{*}$ can be computed as follows

$$
h_{1}^{*}=\frac{K_{1}}{2}\left(\frac{\mu_{b}}{\mu_{g}}\right)=0.25
$$

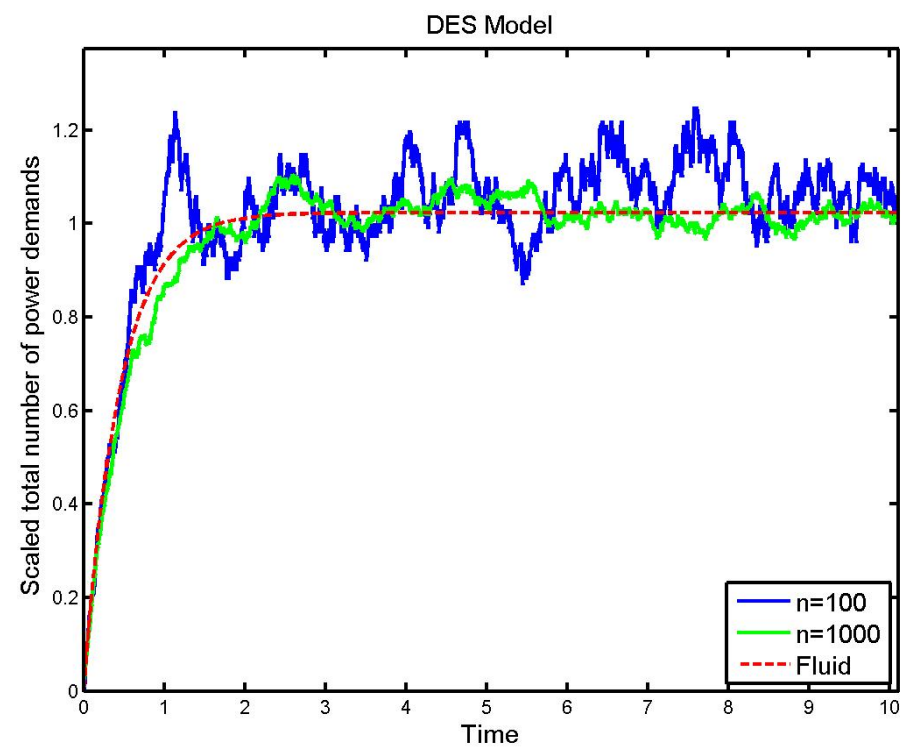

Figure 6.2: A comparison of one sample path of $\bar{X}_{1}^{n}(t)$ to fluid $X_{1}(t)$ of the DES model

Under the policy $h_{1}^{*}$, figure 6.2 shows a comparison of the fluid behaviour of the simulated stochastic power demands in the system at time $t, X_{1}^{n}$, as given in Equation (3.11) to the fluid $X_{1}$ given by Equation (4.1). It is shown that $X_{1}(t)$ bounded by 1. To evaluate the optimal control policy obtained by fluid approximation, a 
comparison of the stochastic total operational cost $\mathcal{P}_{1}^{n, *}(t)$ of Equation (3.4) to the deterministic $\mathcal{P}_{1}^{*}$ of Equation 4.3 is shown in Figure 6.3. In the limit, the stochastic total operational cost under the optimal policy is minimized and the lower bound is achieved as obtained by the deterministic solution proposed in this work, where for this example, $L\left(h_{1}^{*}\right)=0.9994$, and $\mathcal{P}_{1}^{h_{1}^{*}}$ goes to 0.95 in the long run.

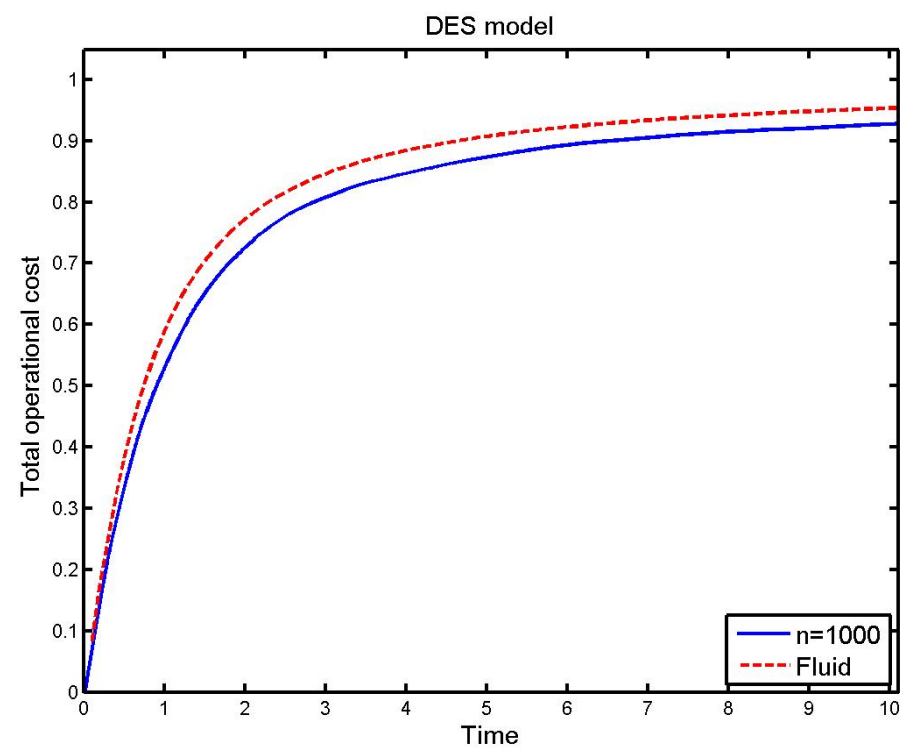

Figure 6.3: A comparison of one sample path and the fluid $\mathcal{P}_{1}^{*}$ of the DES model

\subsection{Simulation Results of the N-DES Model}

A simulation experiment is run for N-DES model described in Section 5.1 where the power demands arrive according to a non-homogenous Poisson process with rate $\lambda(t)=8\left(10 t-t^{2}\right)$, the average total of arrivals is $1 / t\left(\int_{0}^{t} \lambda(s) d s\right)=126.7$, and the average power requirement per demand is $1 \mathrm{~kW}$. The capacity of the grid is $N=150$ and the initial value of the number of power demands in the system, $X_{1}(0)$, is assumed to be zero. Also, the constant $K_{1}=4$, the average service rate by grid is $\mu_{g}=1.2$ power demands/hour, and the average service rate by batteries is $\mu_{b}=0.01$ power demands/hour. Let $n=100$ and apply scaling to find $\bar{\lambda}$ and the process $\Lambda$. Let 
$\psi(h(s))=h_{1}^{2}(s)$. From Equation (5.8),

$$
L\left(\bar{H}_{1}\right):=K_{1} \frac{\bar{\lambda}}{\mu_{g}}-K_{1} \frac{\mu_{b}}{\mu_{g}} \bar{H}_{1}+\left(\bar{H}_{1}^{2}\right)
$$

and

$$
\frac{d L\left(\bar{H}_{1}\right)}{d \bar{H}_{1}}=-K_{1} \frac{\mu_{b}}{\mu_{g}}+2 \bar{H}_{1} .
$$

The optimal capacity of batteries is set to equal to $h_{1}^{*} n$ and $h_{1}^{*}$ can be computed as follows.

$$
h_{1}^{*}=\frac{K 1}{2}\left(\frac{\mu_{b}}{\mu_{g}}\right)=0.0167 .
$$

Under the policy $h_{1}^{*}$, figure 6.4 shows a comparison of the fluid behaviour of the simulated stochastic power demands in the system at time $t, X_{1}^{n}$, as given in Equation (3.11) to the fluid $X_{1}$ given by Equation (5.2). It is shown that $X_{1}(t)$ is bounded by 1.1 .

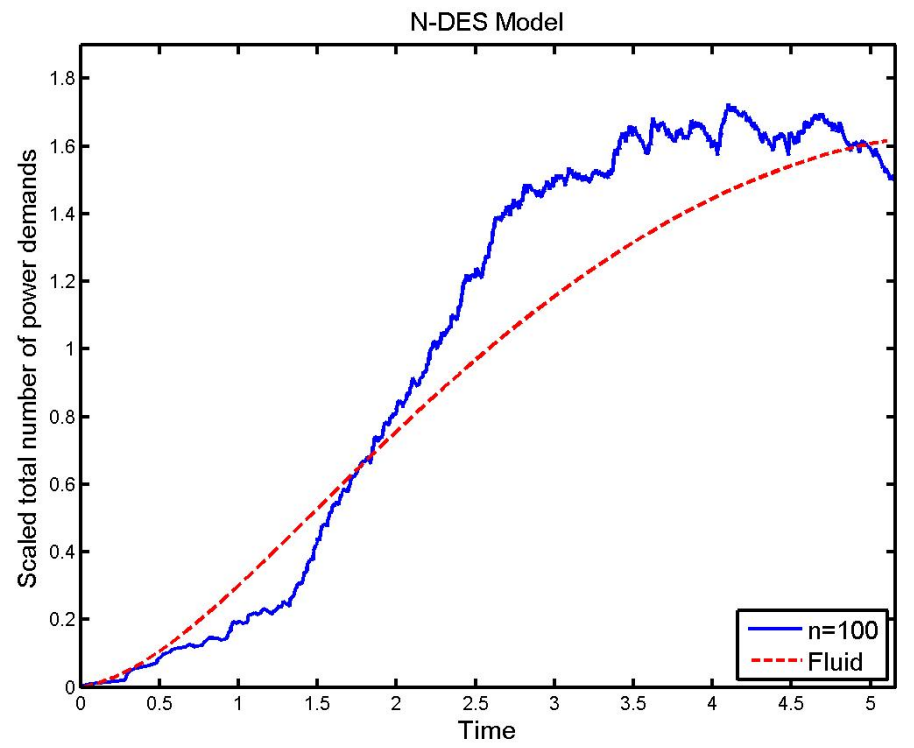

Figure 6.4: A comparison of one sample path of $\bar{X}_{1}^{n}(t)$ to fluid $X_{1}(t)$ of the N-DES model

A comparison of the stochastic total operational cost $\mathcal{P}_{1}^{n, *}(t)$ of Equation (3.4) to the 
deterministic $\mathcal{P}_{1}^{*}$ of Equation (4.3) under the policy $h_{1}^{*}$ is shown in Figure 6.5. In the limit, the stochastic total operational cost under the optimal policy is minimized and achieves the lower bound obtained by the deterministic solution. For this example, $L\left(h^{*}\right)=3.9331$, and $\mathcal{P}_{1}^{h_{1}^{*}}$ goes to 3.7639 .

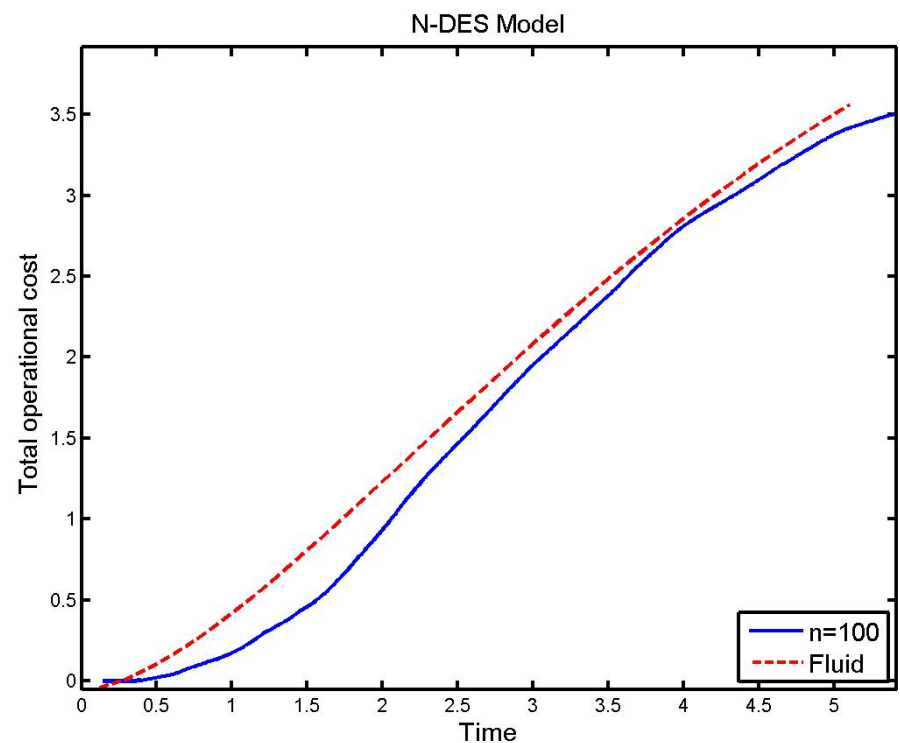

Figure 6.5: A comparison of one sample path and the fluid $\mathcal{P}_{1}^{*}$ of the N-DES model

\subsection{Simulation Results of the RES Model}

Another simulation experiment is run to validate the fluid approximation of the smart grid for RES model described in Section 5.2. In this experiment, the grid capacity is suppose to be infinity, but it is represented by $N=2200$. The arrival rate $\lambda n=2 n$ power demands/hour is set to be very large. where $n=200$ and $n=1000$. The initial value of the number of power demands, $X_{2}(0)$, is assumed to be zero and the constants $K_{1}=2, K_{2}=1.5$. The service rates from grid and batteries are $\mu_{g}=2.5$ power demands/hour, $\mu_{b}=0.5$ power demands/hour respectively, and the recharging rate from grid $\alpha=2$ empty batteries/hour. let the average power requirement per 
demand is $1 \mathrm{~kW}$, and $C\left(h_{2}(s)\right)=h_{2}^{2}(s)$. From Equation (5.32),

$$
L\left(\bar{H}_{2}\right):=K_{1} \frac{\lambda}{\mu_{g}}-K_{1} \frac{\mu_{b}}{\mu_{g}} \bar{H}_{2}+\frac{\mu_{b} K_{2}}{\alpha} \bar{H}_{2}+\left(\bar{H}_{2}^{2}\right)
$$

and

$$
\frac{d L\left(\bar{H}_{2}\right)}{d \bar{H}_{2}}=-K_{1} \frac{\mu_{b}}{\mu_{g}}+\frac{\mu_{b} K_{2}}{\alpha}+2 \bar{H}_{2} .
$$

The optimal capacity of batteries is set to equal to $h_{2}^{*} n$ and $h_{2}^{*}$ can be computed as follows.

$$
h_{2}^{*}=\frac{1}{2}\left(\frac{K_{1} \mu_{b}}{\mu_{g}}-\frac{K_{2} \mu_{b}}{\alpha}\right)=0.0125
$$

Additionally, in this simulation experiment of the RES model, the initial capacity of the batteries $B(0)$ is set to be equal $h_{2}^{*} n$. Figure 6.6 shows a comparison of the fluid behaviour of the simulated stochastic power demands in the system at time $t, X_{2}^{n}$ given by Equation (5.19), to the fluid $X_{2}$ given by Equation (5.21). It is shown that $X_{2}(t)$ bounded by 0.81 .

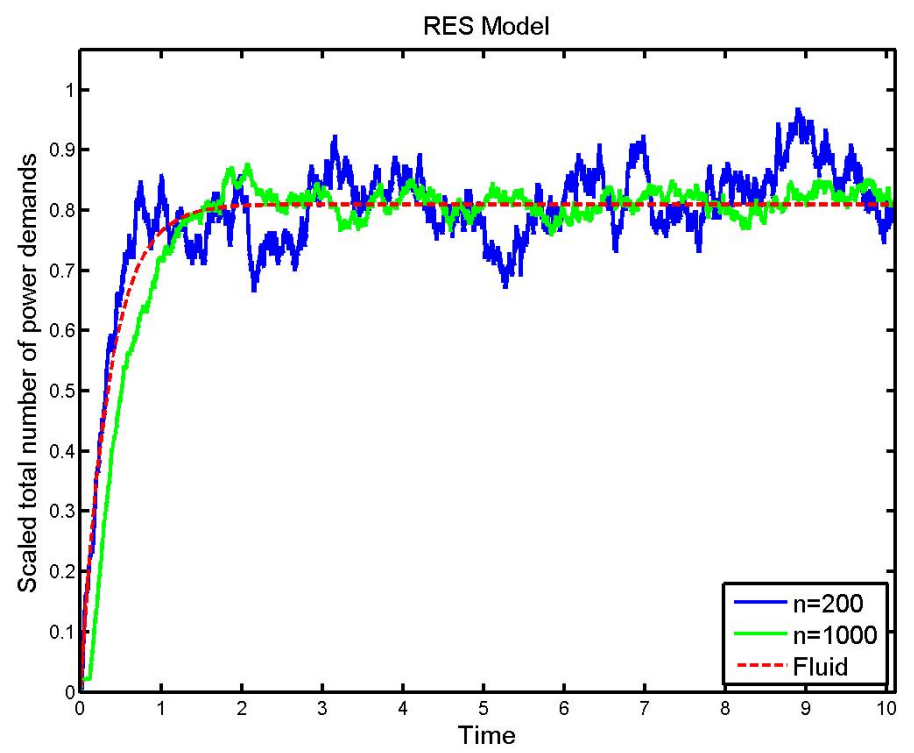

Figure 6.6: A comparison of one sample path of $\bar{X}_{2}^{n}(t)$ to fluid $X_{2}(t)$ of the RES model

To evaluate the optimal control policy obtained by fluid approximation, a comparison of the stochastic total operational $\operatorname{cost} \mathcal{P}_{2}^{n, *}(t)$ given by Equation (5.10) to 
the deterministic total operational cost $\mathcal{P}_{2}^{*}$ given by Equation (5.24) is shown in Figure 6.7. In the limit, the stochastic total operational cost under the optimal policy is minimized and achieves the lower bound obtained by the deterministic solution proposed in this work, where for this example, $L\left(h^{*}\right)=1.5998$, and $\mathcal{P}_{2}^{h^{*}}$ goes to 1.55 .

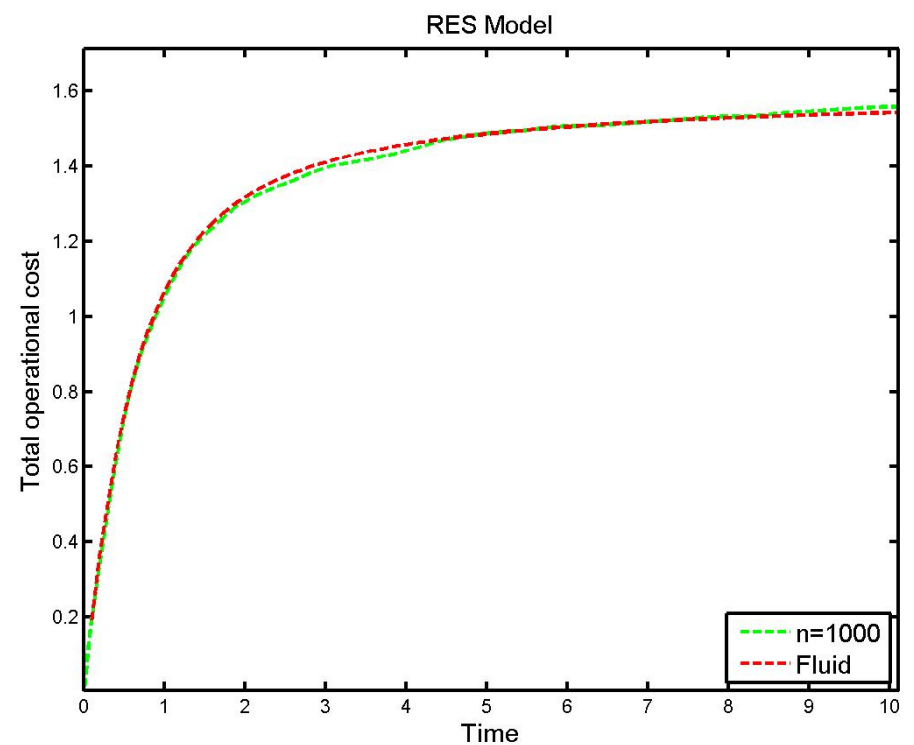

Figure 6.7: A comparison of one sample path and the fluid $\mathcal{P}_{2}^{*}$ of the RES model

From the previous given examples for each model, it can be observed that the optimal control policy works for different values of $\lambda, \mu_{g}, \mu_{b}, \alpha, K_{1}$, and $K_{2}$. However, a careful selection of them should be made in each case.

\subsection{More Simulation Results of the Smart Grid}

In this section, another two experiments are run to compare between the total operational cost in case of the proposed models of the smart grid with batteries and the total operational cost in case of the smart grid without batteries. The simulation results show that the addition of the batteries in each model did not change the cost that much, but the system was allowed to accept more power demands. These results are shown in Figures 6.8 and 6.9 . 


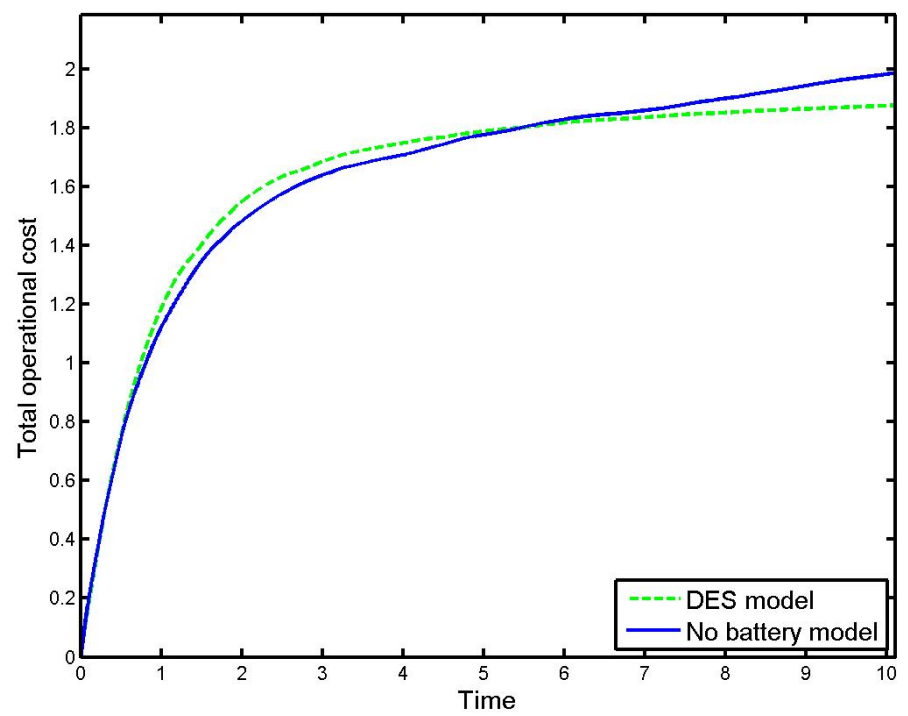

Figure 6.8: A comparison of the cost $\mathcal{P}_{1}^{h_{1}^{*}}$

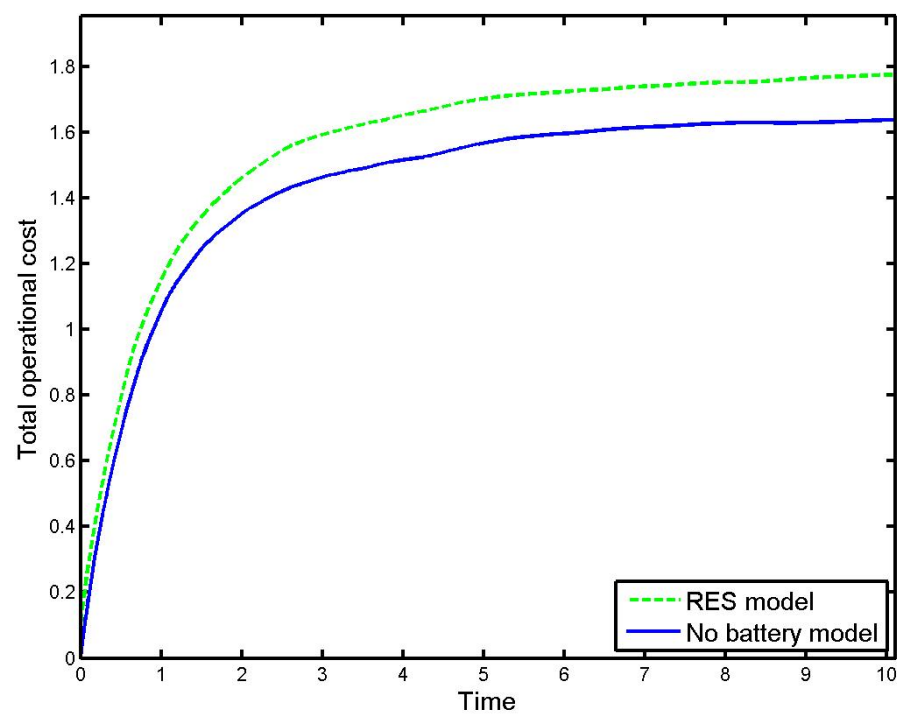

Figure 6.9: A comparison of the cost $\mathcal{P}_{2}^{h_{2}^{*}}$ 


\section{Chapter 7}

\section{Conclusion and Future Work}

The main goal of this work is to manage the capacity of batteries so that the total operational cost of the smart grid with batteries is minimized. The applied fluid approximation method was able to turn the difficult stochastic optimal control problem into a much easier deterministic control problem. The solution of deterministic problem provides a simple control policy for the pre-limit stochastic model, which is shown to be asymptotically optimal. Also, solving the deterministic optimization problem by fluid approximation provides a good alternative to numerical methods such as Markov Decision Processes.

One can also look beyond deterministic fluid approximation, to obtain a diffusion process refinement. Many open problems remain. For example, corresponding performance approximations for the system with non-exponential distributions can be developed. Additionally, the discussion was limited to heavy load conditions where the arrival rate is very large, but the control and the results could be extended to other regimes and more general systems. Such extensions remain a topic for future research. 


\section{List of References}

[1] I. Koutsopoulos, V. Hatzi, and L. Tassiulas, "Optimal energy storage control policies for the smart power grid," in Smart Grid Communications (SmartGridComm), 2011 IEEE International Conference on, pp. 475-480, IEEE, 2011.

[2] R. Atar, C. Giat, and N. Shimkin, "The c/ rule for many-server queues with abandonment," Operations Research, vol. 58, no. 5, pp. 1427-1439, 2010.

[3] P. W. Sauer, "Educational needs for the smart grid workforce," in Power and Energy Society General Meeting, 2010 IEEE, pp. 1-3, IEEE, 2010.

[4] I. S. Bayram, G. Michailidis, M. Devetsikiotis, F. Granelli, and S. Bhattacharya, "Smart vehicles in the smart grid: Challenges, trends, and application to the design of charging stations," in Control and Optimization Methods for Electric Smart Grids, pp. 133-145, Springer, 2012.

[5] R. Urgaonkar, B. Urgaonkar, M. J. Neely, and A. Sivasubramaniam, "Optimal power cost management using stored energy in data centers," in Proceedings of the ACM SIGMETRICS joint international conference on Measurement and modeling of computer systems, pp. 221-232, ACM, 2011.

[6] M. Gatzianas, L. Georgiadis, and L. Tassiulas, "Control of wireless networks with rechargeable batteries [transactions papers]," Wireless Communications, IEEE Transactions on, vol. 9, no. 2, pp. 581-593, 2010.

[7] O. Perry and W. Whitt, "A fluid approximation for service systems responding to unexpected overloads," Operations research, vol. 59, no. 5, pp. 1159-1170, 2011.

[8] M. M. Karbasioun, I. Lambadaris, G. Shaikhet, and E. Kranakis, "Optimal charging strategies for electrical vehicles under real time pricing," in Proceedings of 2014 IEEE International Conference on Smart Grid Communications: "Demand Response and Dynamic Pricing, 2014.

[9] W. L. Winston, Operations Research, Applications and Algorithms, 3rd Edition. Duxbury Press, third ed., 1994.

[10] O. C. Ibe, Fundamentals of stochastic networks. John Wiley \& Sons, 2011. 
[11] H. Chen and D. D. Yao, "Fundamentals of queuing networks," 2001.

[12] G. F. Newell, Applications of queueing theory, [by] G. F. Newell. Chapman and Hall London, 1971.

[13] A. R. Ward, "Asymptotic analysis of queueing systems with reneging: A survey of results for fifo, single class models," Surveys in Operations Research and Management Science, vol. 17, no. 1, pp. 1 - 14, 2012.

[14] R. B. Cooper, "Introduction to queueing theory," 1972.

[15] N. S. Wade, P. Taylor, P. Lang, and P. Jones, "Evaluating the benefits of an electrical energy storage system in a future smart grid," Energy policy, vol. 38, no. 11, pp. 7180-7188, 2010.

[16] S. M. Ross, A course in simulation. Prentice Hall PTR, 1990.

[17] G. Birkhoff and G.-C. Rota, Ordinary Differential Equations, 4th Edition. Wiley, fourth ed., Jan. 1989. 


\section{Appendix A}

\section{Some Useful Theorems}

Theorem A.1. (Functional Law of Large Number) Let $\left\{a_{n}\right\}$ be a sequence of positive IID random variables having a finite mean $1 / r>0$. If we define the renewal process $R(t)$ where $R(t)=\sup \left\{n: a_{n} \leq t\right\}$, for $T>0$. With probability 1 , $\sup _{t \in[0, T]}\left|\frac{1}{n} A^{n}(t)-\lambda t\right| \longrightarrow 0$ as $n \rightarrow \infty$.

Theorem A.2. (Jensen's inequality) For a convex function $\psi$ and a random variable $Y$ we have $E[\psi(Y)] \geq \psi[E(Y)]$.

As a result, for any function $h_{1}$ whose support includes $[0, t]$, we must have

$$
\frac{1}{t} \int_{0}^{t} \psi\left(h_{1}(s)\right) d s \geq \psi\left(\frac{1}{t} \int_{0}^{t} h_{1}(s) d s\right)
$$

Indeed, the integral on the l.h.s. can be represented as an expectation of a convex function of the random variable $Y:=h_{1}(S)$, where $S$ is uniform on $[0, t]$. And the relation A.1 simply follows from Jensen inequality.

Theorem A.3. (Comparison theorem) [17] Let $f$ and $g$ be solutions of the differential equations $\frac{d X(t)}{d t}=F_{1}(t, X(t)), \frac{d Y(t)}{d t}=F_{2}(t, Y(t))$, respectively, satisfying the same initial conditions $f(a)=g(a)$. Suppose further that $F_{1}$ and $F_{2}$ satisfy a Lipschitz condition, and that $F_{1}(t, u) \leq F_{2}(t, u)$ for all $t$ and $u$ in a given domain. Then, $f \leq g$ for $t>a$. 


\section{Appendix B}

\section{Code for Simulations}

\section{B.1 Simulation Programs of $M / M / N$ Queuing Model}

\%

$\%$ This is a simulation program of an $\mathrm{M} / \mathrm{M} / \mathrm{N}$ queueing model $\%$

$\%$ under heavy load conditions. It is assumed that this is $\%$

$\%$ an initially empty system. The mean service time is 1 and $\%$

$\%$ the observation time is $[0,10.1]$. $\%$

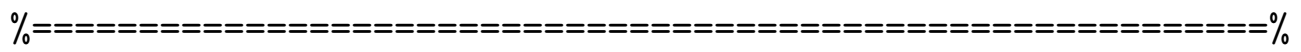

function M_M_N_J(n, color)

$\%$ M/M/N Queueing Network Simulation program:

$\mathrm{T}=10.1$;

Lamda $=0.8 * \mathrm{n}, \%$ then Lamda $=6000$ and it is the arrival rate.

$\mathrm{N}=3 * \mathrm{n}, \% \mathrm{~N}$ is the number of servers

$\mathrm{M}=1 ; \% \mathrm{M}$ is the service rate.

$\mathrm{X}=\operatorname{zeros}(1,101)$;

$\mathrm{w}=1$;

time $=[]$;

time $=[$ time 0$]$;

$\mathrm{X}=[]$; 


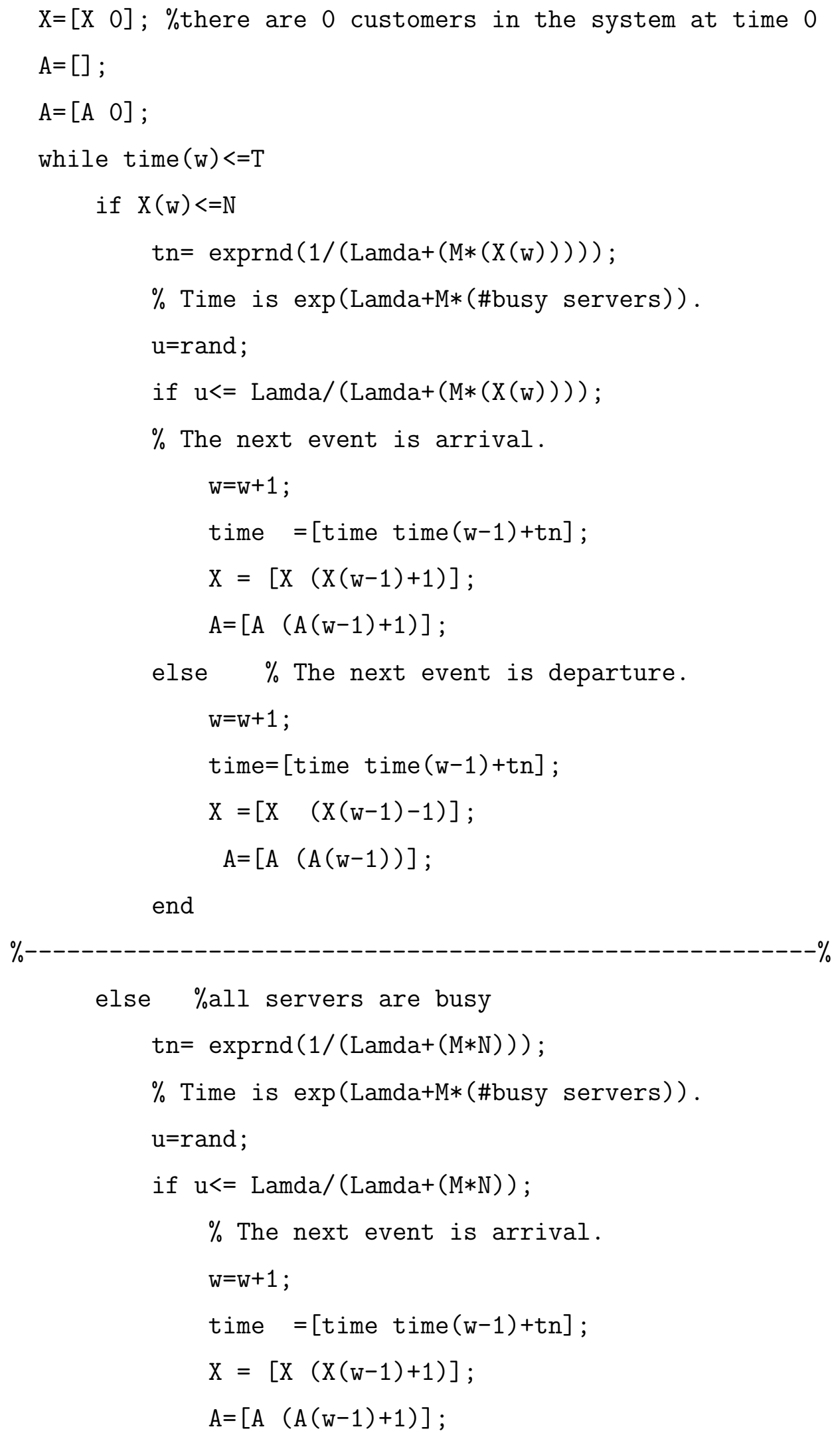


else $\%$ The next event is departure.

$$
\begin{aligned}
& \mathrm{W}=\mathrm{W}+1 ; \\
& \text { time }=[\text { time time }(\mathrm{w}-1)+\mathrm{tn}] \\
& \mathrm{X}=[\mathrm{X} \quad(\mathrm{X}(\mathrm{W}-1)-1)] \\
& \mathrm{A}=[\mathrm{A}(\mathrm{A}(\mathrm{W}-1))] ;
\end{aligned}
$$

end

end

end

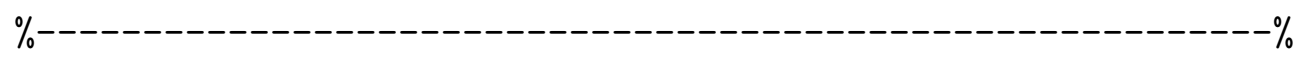

$j=1$;

X_bar $=\mathrm{X} . / \mathrm{n}$;

plot (time, X_bar, 'Color', color, 'LineWidth', 2)

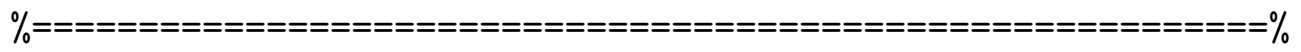

$\%$ This program creates deterministically a plot of the num- $\%$

$\%$ ber of jobs in the $M / M / N$ system, $Q$. $\%$

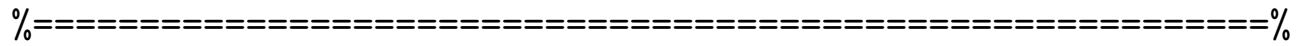

$\mathrm{t}=0$;

lamda=0.8; \% Arrival rate.

$\mathrm{M}=1 ; \quad \%$ service rate.

$\mathrm{n}=1$;

$\mathrm{Q}=[]$;

$\mathrm{Q}=\left[\begin{array}{ll}\mathrm{Q} & 0\end{array}\right]$;

$d t=0.1$;

$\mathrm{T}=[]$;

$\mathrm{T}=\left[\begin{array}{ll}\mathrm{T} & \mathrm{t}\end{array}\right]$;

while $\mathrm{T}(\mathrm{n})<10$

$\mathrm{n}=\mathrm{n}+1$;

$t=t+d t$;

$\mathrm{q}=(\mathrm{Q}(\mathrm{n}-1)+1$ amda $* d t)-(M *(\min (\mathrm{Q}(\mathrm{n}-1), 1)) * d t) ;$ 
$\mathrm{Q}=\left[\begin{array}{ll}\mathrm{Q} & \mathrm{q}\end{array}\right]$;

$\mathrm{T}=\left[\begin{array}{ll}\mathrm{T} & \mathrm{t}\end{array}\right]$;

end

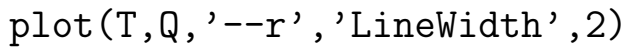

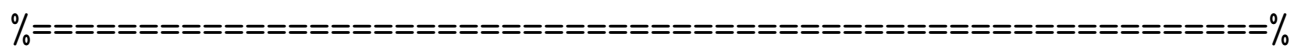

$\%$ This program compares between the fluid behaviour of the $\%$ $\%$ simulated stochastic total number of jobs in the system $\%$ $\%$ Q_bar and the deterministic Q. $\%$

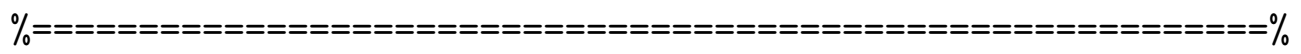

M_M_N_J (100, 'blue')

hold on;

M_M_N_J (1000, 'green')

M_M_N_fluid_J ;

hold off;

hleg=legend ('n=100', 'n=1000', 'Fluid') ;

set (hleg, 'Location', 'SouthEast', 'FontSize', 14);

title('M//M/N Queuing Model','FontSize',14);

xlabel ('Time', 'fontsize', 14);

ylabel('Scaled total number of customers','fontsize',14);

set (gca, 'LineWidth', 2);

axis tight;

axis_val=axis;

axis_val=...

[axis_val(1) axis_val(2) axis_val(3)*1.1 axis_val(4)*1.1] ;

$\operatorname{axis}($ axis_val);

\section{B.2 Simulation Programs of DES Model}

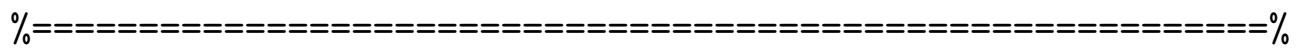


$\%$ This is the DES model simulation program. In this example\% $\%$ the optimal policy is tested where the total number of $\%$ $\%$ power demands that can be served by batteries is fixed to\% $\%$ h_star. There are infinite servers represented by $\mathrm{N}$ and $\%$ $\%$ empty batteries get replaced with full ones immediately. $\%$ $\%$ It is assumed that this is an initially empty system. Po-\% $\%$ wer demands arrive with very big rate Lamda and are serv-\% $\%$ ed by batteries and grid with service times of means $\%$ $\% 1 / M_{-} b$ and 1/M_g respectively. The observation time is $\%$ $\%[0,10.1]$. $\mathrm{X} 1$ is the scaled total number of power demands $\%$ $\%$ in the system. P1_star is the optimal total operational \% $\%$ cost given the optimal policy h_star. $\%$

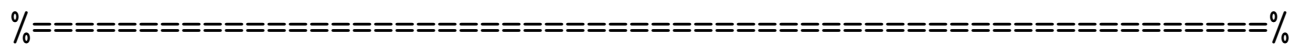

\%function DES_Model_J(N,color)

$\mathrm{N}=2.2 * \mathrm{n} ; \quad \%$ Grid capacity .

$\mathrm{T}=10.1$;

$\mathrm{k} 1=1$;

M_g=2; $\quad \%$ M_g is the service rate from grid.

M_b $=0.1 ; \% M_{-} b$ is the service rate from battery. Lamda $=2 * n ; \%$ Arrival rate.

h_star $=(\mathrm{k} 1 / 2) *\left(M_{-} \mathrm{b} / M_{-} g\right) \%$ The optimal policy. battery_size=h_star $*$ n;

$\mathrm{W}=1$;

$\mathrm{X} 1=[]$;

time $=[]$;

time $=[$ time 0$]$;

$\mathrm{A}=[]$;

$\mathrm{A}=\left[\begin{array}{ll}\mathrm{A} & 0\end{array}\right]$;

$\mathrm{B}=[]$;

$\mathrm{B}=[\mathrm{B}$ battery_size $]$; \% Battery max capacity. $\mathrm{Xg}=[]$; 
$\% \mathrm{Xg}$ is the number of power demands being served by the grid. $\mathrm{h}=[]$;

$\% \mathrm{~h}$ is the number of power demands being served by Battery. $\mathrm{Xg}=\left[\begin{array}{ll}\mathrm{Xg} & 0\end{array}\right]$;

$\%$ Initial number of power demands being served by the grid. $\mathrm{h}=\left[\begin{array}{ll}\mathrm{h} & 0\end{array}\right]$;

P1_star=[];

P1_star $=\left[P 1 \_s t a r 0\right]$;

while time $(W)<=T$

if $h(W)<B(1) \%$ Free servers at the battery.

tdb=inf; \% tdb is the departure time from battery. ta $=\operatorname{exprnd}(1 /$ Lamda);

$\%$ ta is the arrival time and it is exp(Lamda).

$\mathrm{W}=\mathrm{W}+1$;

time $=[$ time time $(W-1)+$ ta $]$;

$\mathrm{Xg}=[\mathrm{Xg} \mathrm{Xg}(\mathrm{W}-1)] ;$

$A=[A \quad A(W-1)+1]$;

$\mathrm{h}=[\mathrm{h}(\mathrm{h}(\mathrm{W}-1)+1)]$;

$\%$

else $h(W)==B(1)$

if $\mathrm{Xg}(\mathrm{W})<\mathrm{N}$

$\%$ Power demands can be served from grid now.

ta $=\operatorname{exprnd}(1 /$ Lamda) $\%$ Generate arrival time.

$\operatorname{tdb}=\operatorname{exprnd}\left(1 /\left(M_{-} b * h(W)\right)\right)$;

if $\mathrm{Xg}(\mathrm{W})==0$

$\operatorname{tdg}=$ inf ;

else

$\operatorname{tdg}=\operatorname{exprnd}\left(1 /\left(\mathrm{Xg}(\mathrm{W}) * \mathrm{M}_{-} \mathrm{g}\right)\right) ;$

end

if $t a<=\min (t d g, t d b) \%$ The next event is arrival. $\mathrm{W}=\mathrm{W}+1$; 


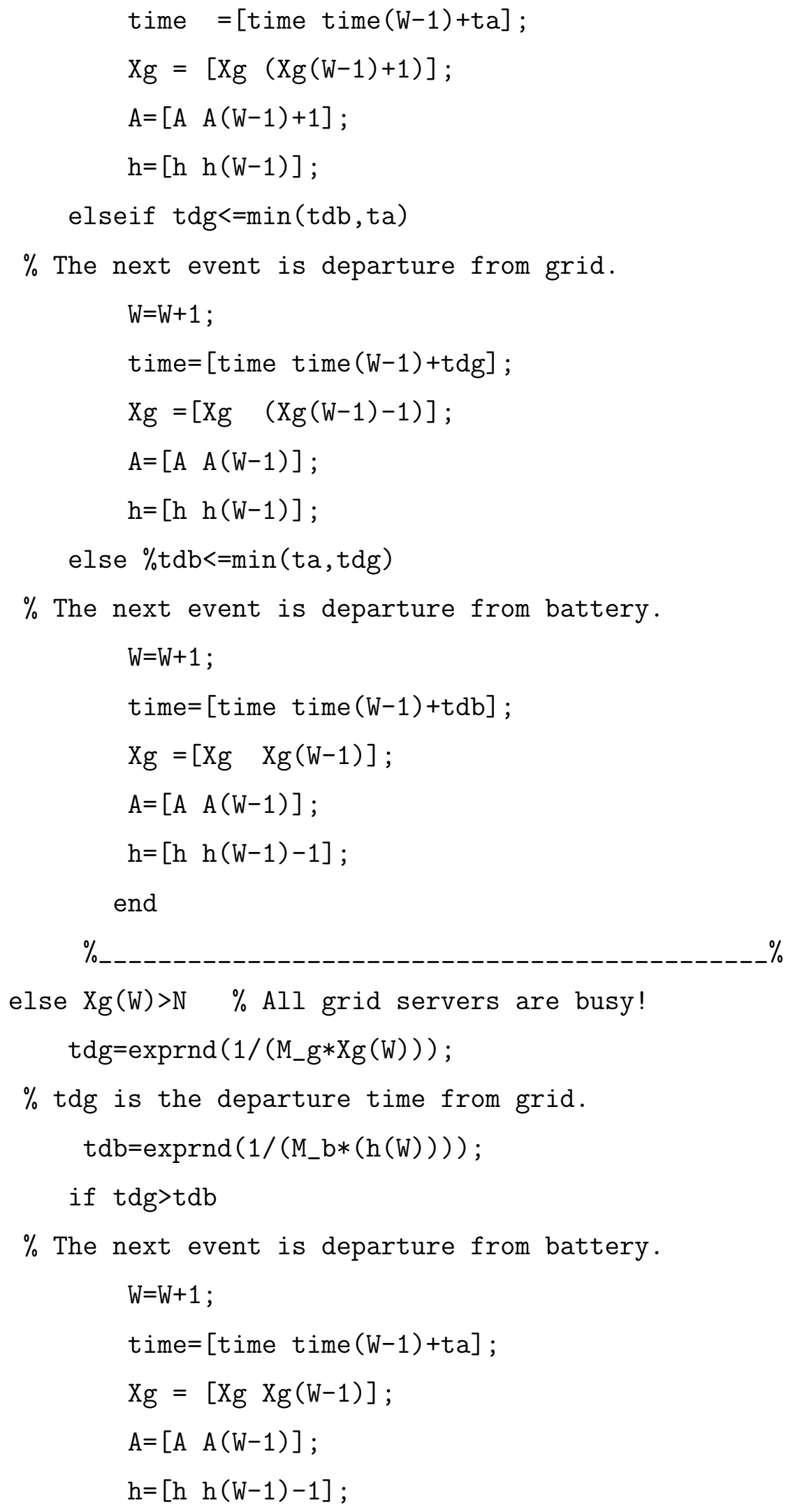




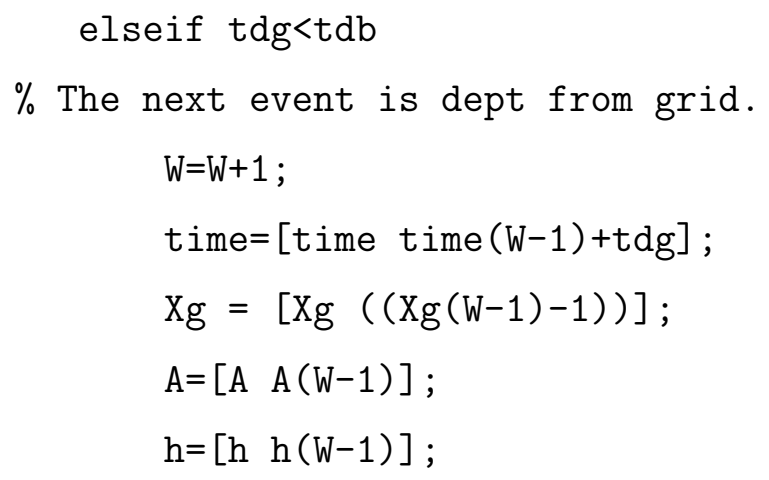


$\% \quad P 1 \_s t a r=k 1 *\left(X_{g}\right.$ _int2./time $)+h \_i n t c . / t i m e ;$

\% plot(time,P1_star, 'b', 'LineWidth', 2)

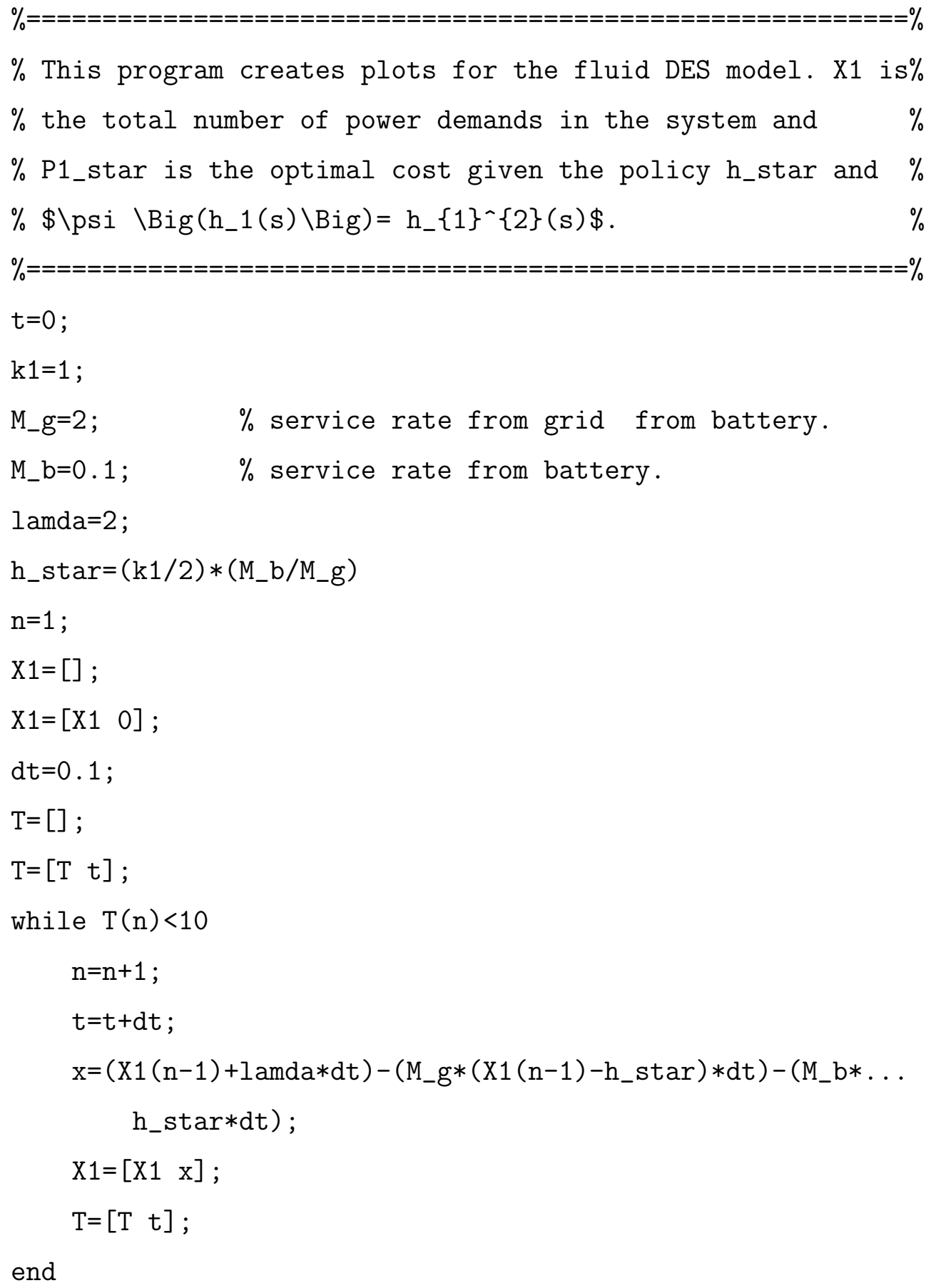




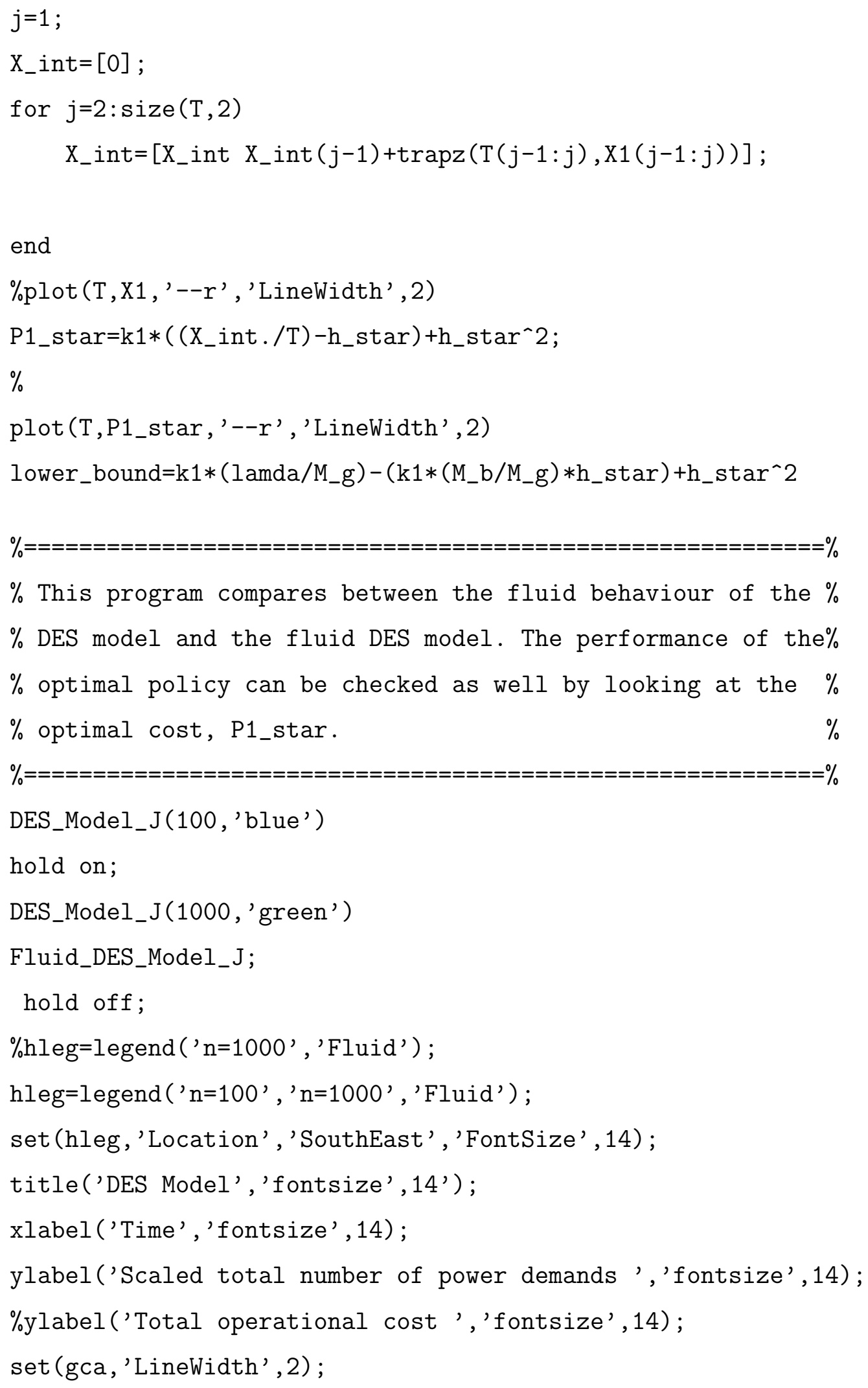


axis tight;

axis_val=axis;

axis_val=...

[axis_val(1) axis_val(2) axis_val(3)*1.1 axis_val(4)*1.1] ;

axis(axis_val);

\section{B.3 Simulation Programs of the N-DES Model}

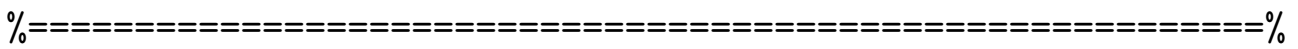

$\%$ This is the N-DES model simulation program. In this exam-\%

$\%$ ple the optimal policy is tested where the total number \%

$\%$ of power demands that can be served by batteries is fixed\%

$\%$ to $\mathrm{h}_{-}$star. Power demands arrive according to nonhomogeno- $\%$

$\%$ ues Poisson process with big rate and are served by batt- $\%$

$\%$ eries and grid with exponentially distributed times of $\%$

$\%$ means $1 / M_{-} b$ and $1 / M_{-} g$ respectively. There are $\mathrm{N}$ servers $\%$

$\%$ in total and empty batteries are assumed to be replaced \%

$\%$ with full ones immediately. $\%$

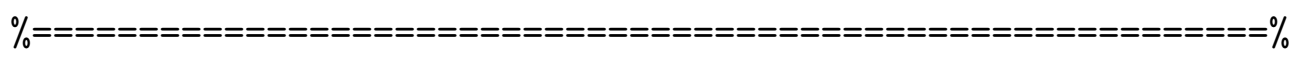

$\%$ To generate the first $\mathrm{T}$ unites of nonhomogenoues

$\%$ Piosson arrivals,

$\mathrm{T}=[]$;

$\mathrm{t}=0$;

next_arrival $=0$;

lamda_max $=200 ; \%$ the maximum of Lamda( $t)$ at time $t=5$

stop $=0$;

while stop $=0$

done $=0$;

while done $==0$

$\mathrm{u} 1=\mathrm{rand}$;

$\mathrm{t}=\mathrm{t}-\left(1 / \mathrm{lamda} \_\max \right) * \log (\mathrm{u} 1)$; 


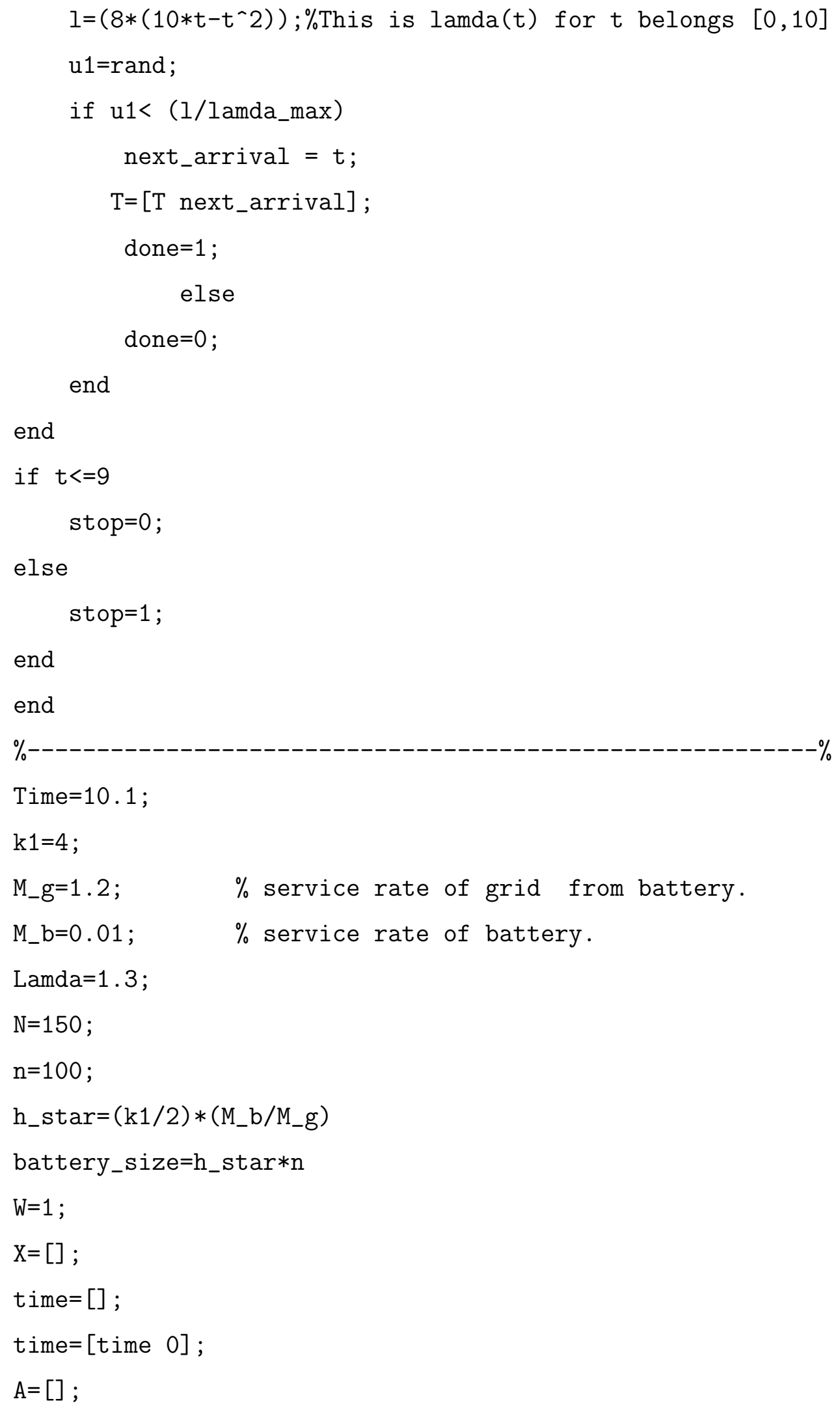


$\mathrm{A}=\left[\begin{array}{ll}\mathrm{A} & 0\end{array}\right]$;

$\mathrm{B}=[]$;

$\mathrm{B}=[\mathrm{B}$ battery_size $]$; \% Battery level

$\mathrm{Xg}=[]$;

$\% \mathrm{Xg}$ is the number of power demands being served by grid.

$\mathrm{h}=[]$;

$\% \mathrm{~h}$ is the number of power demands being served by battery. $\mathrm{Xg}=\left[\begin{array}{ll}\mathrm{Xg} & 0\end{array}\right]$;

$\mathrm{h}=\left[\begin{array}{ll}\mathrm{h} & 0\end{array}\right]$;

$\%$ It is assumed that this is an initially empty system.

while $\mathrm{W}<=1250$

if $\mathrm{h}(\mathrm{W})<\mathrm{B}(1) \%$ Free servers at the battery.

tdb=inf; $\%$ tdb is the departure time from battery.

ta $=\mathrm{T}(\mathrm{W}) ; \%$ generate arrival time

$\mathrm{W}=\mathrm{W}+1$;

time $=[$ time time $(W-1)+$ ta $]$;

$\mathrm{Xg}=[\mathrm{Xg} \mathrm{Xg}(\mathrm{W}-1)]$;

$A=[A \quad A(W-1)+1]$;

$h=[h(h(W-1)+1)]$;

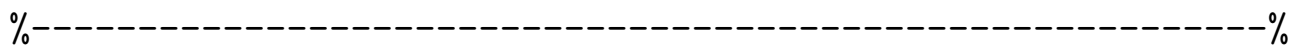

else $h(W)==B(1)$

if $\mathrm{Xg}(\mathrm{W})<\mathrm{N}$

$\%$ Power demands can be served from grid now.

ta $=\mathrm{T}(\mathrm{W})-\mathrm{T}(\mathrm{W}-1) ; \%$ Generate arrival time.

$\operatorname{tdb}=\operatorname{exprnd}\left(1 /\left(M_{-} \mathrm{b} * \mathrm{~h}(\mathrm{~W})\right)\right)$;

if $\mathrm{Xg}(\mathrm{W})==0$

$\operatorname{tdg}=$ inf ;

else

$\operatorname{tdg}=\operatorname{exprnd}\left(1 /\left(\mathrm{Xg}_{(\mathrm{W})} * \mathrm{M}_{-} \mathrm{g}\right)\right) ;$

end

if $t a<=\min (t d g, t d b) \%$ The next event is arrival. 


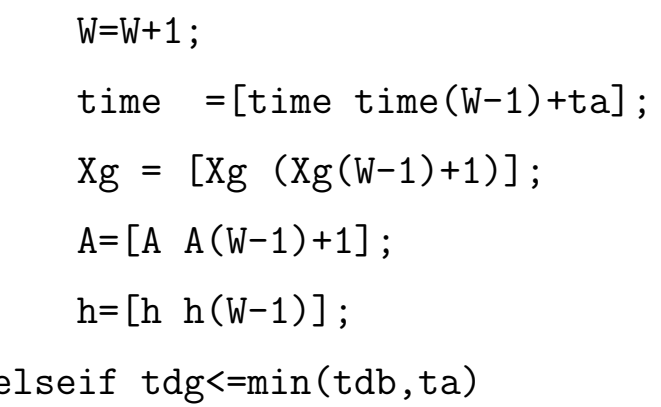

$\%$ The next event is departure from grid.

$\mathrm{W}=\mathrm{W}+1$;

time $=[$ time time $(W-1)+$ tdg $]$;

$\mathrm{Xg}=\left[\begin{array}{ll}\mathrm{Xg} & (\mathrm{Xg}(\mathrm{W}-1)-1)\end{array}\right]$;

$A=[A \quad A(W-1)]$;

$h=[h h(W-1)]$;

else $\%$ tdb $<=\min (t a, t d g)$

$\%$ The next event is departure from battery.

$\mathrm{W}=\mathrm{W}+1$;

time $=[$ time time $(W-1)+t d b]$;

$\mathrm{Xg}=[\mathrm{Xg} \quad \mathrm{Xg}(\mathrm{W}-1)]$;

$A=[A(W-1)]$;

$\mathrm{h}=[\mathrm{h} h(\mathrm{~W}-1)-1]$;

end

$\%$

else $\mathrm{Xg}(\mathrm{W})>\mathrm{N} \quad \%$ All grid servers are busy! $\operatorname{tdg}=\operatorname{exprnd}\left(1 /\left(M_{-} g * \operatorname{Xg}(W)\right)\right)$;

$\%$ tdg is the deptrture time from grid.

$t d b=\operatorname{exprnd}\left(1 /\left(M_{-} b *(h(W))\right)\right)$;

if $\mathrm{tdg}>\mathrm{tdb}$

$\%$ The next event is departure from battery.

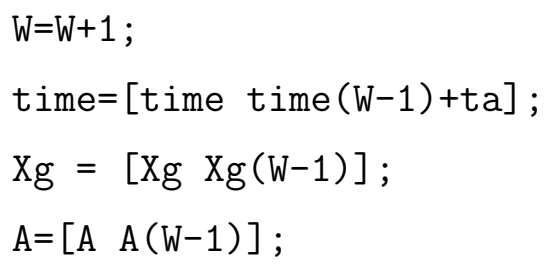




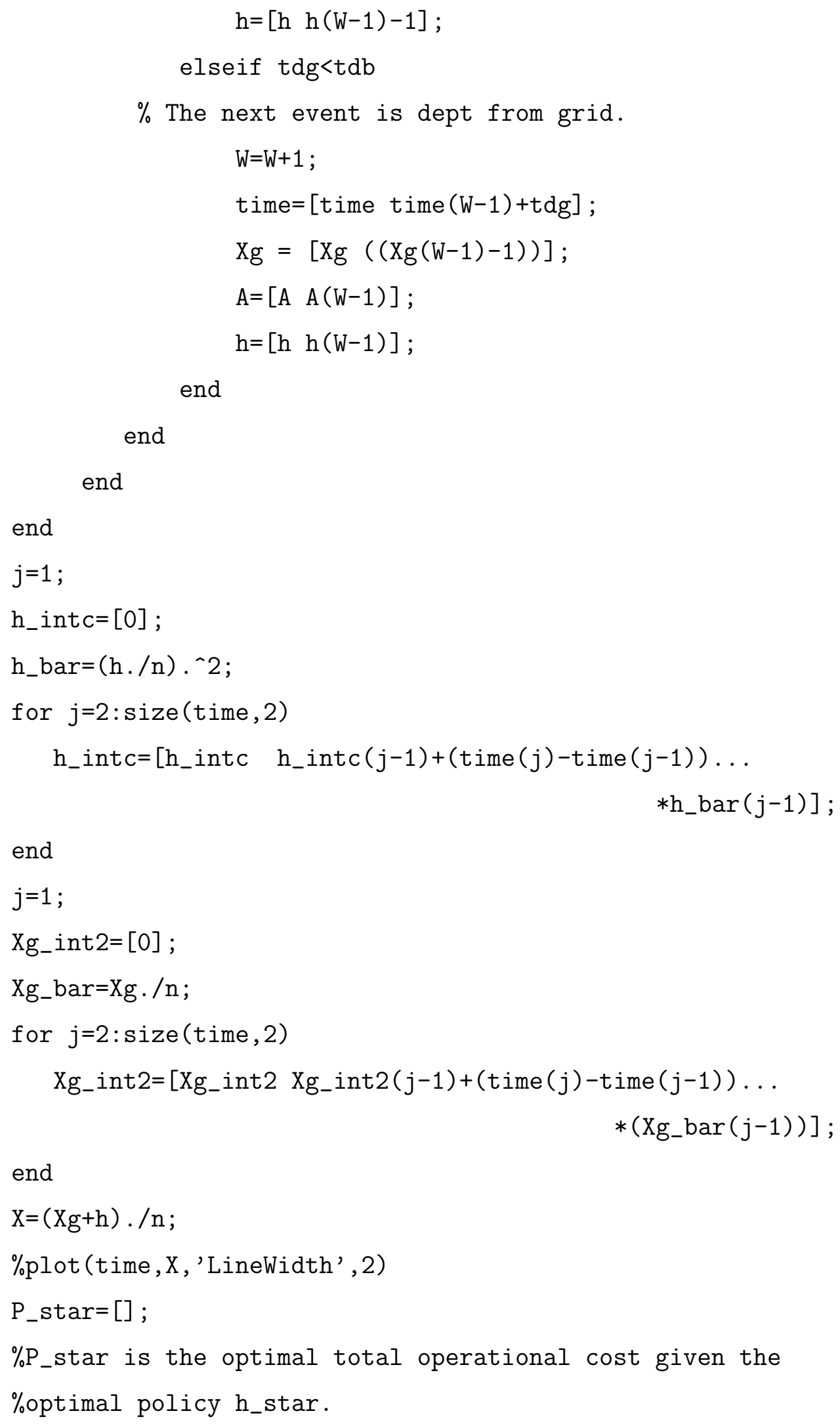




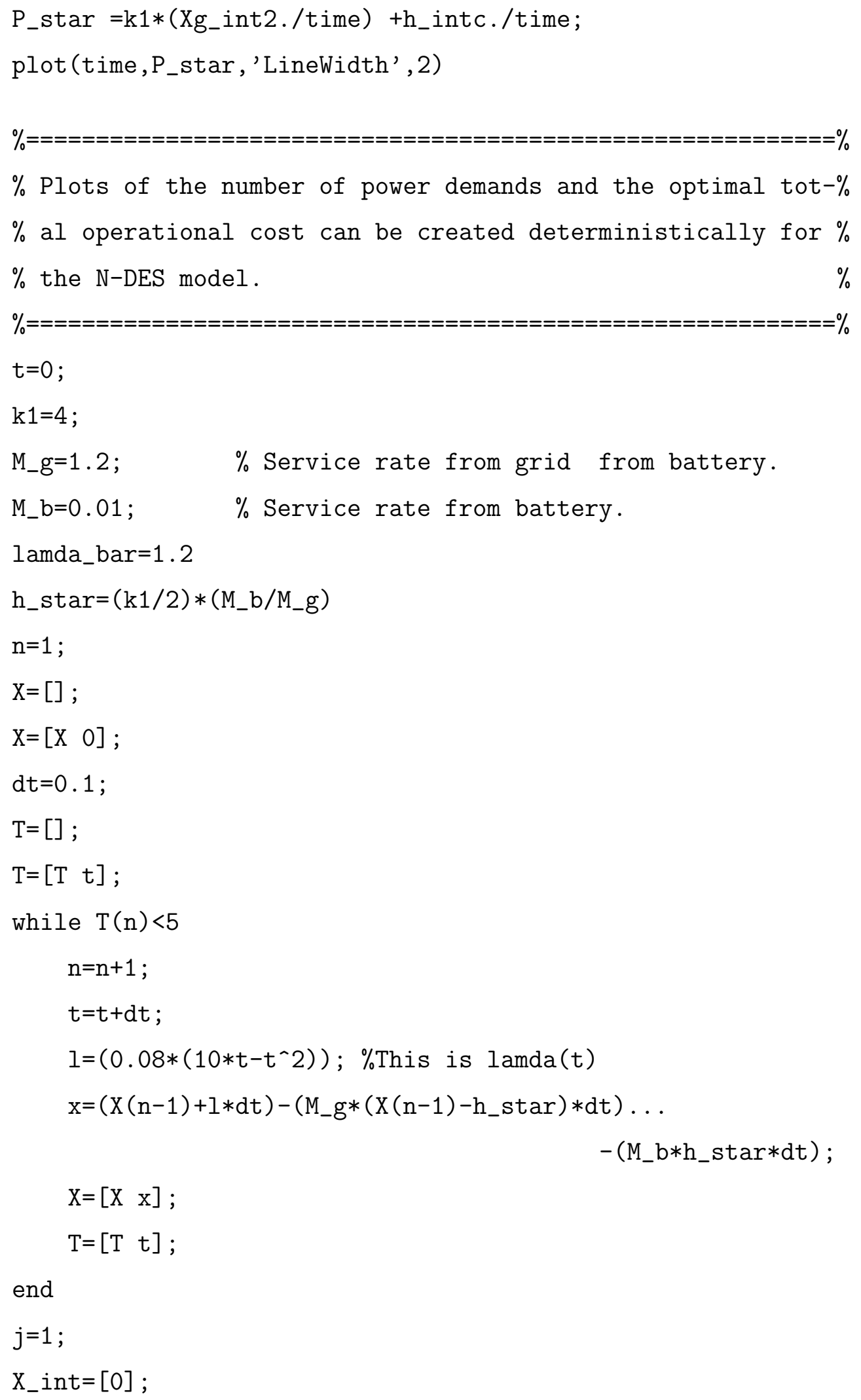


for $\begin{aligned} & j=2: \operatorname{size}(T, 2) \\ & X_{-} \text {int }=\left[X_{-} \text {int } X_{-} \operatorname{int}(j-1)+\operatorname{trapz}(T(j-1: j), X(j-1: j))\right] ;\end{aligned}$

end

\% $\operatorname{plot}(\mathrm{T}, \mathrm{X}$, , $--\mathrm{r}$ ', 'LineWidth', 2)

lower_bound=k1*(lamda_bar/M_g $)-\left(\mathrm{k} 1 *\left(M_{-}\right.\right.$b/M_g $) *$ h_star $)+h_{-} s t a r^{\wedge} 2$

$\mathrm{P}=\mathrm{k} 1 *\left(\left(\mathrm{X}_{-}\right.\right.$int./T) $\left.-\mathrm{h}_{\text {_star }}\right)+\mathrm{h}_{\text {s }}$ star^2 2 ;

plot (T, P, '--r', 'LineWidth', 2)

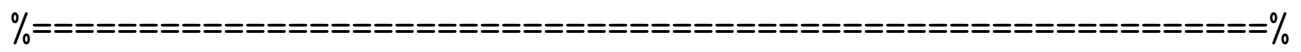

$\%$ This program compares between the fluid behaviour of the $\%$ $\%$ N-DES model and the deterministic N-DES model. The perfo- $\%$ $\%$ rmance of the optimal policy can be checked as well by $\%$ $\%$ looking at the plots of the optimal cost, P1_star. \%

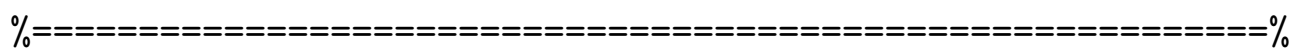

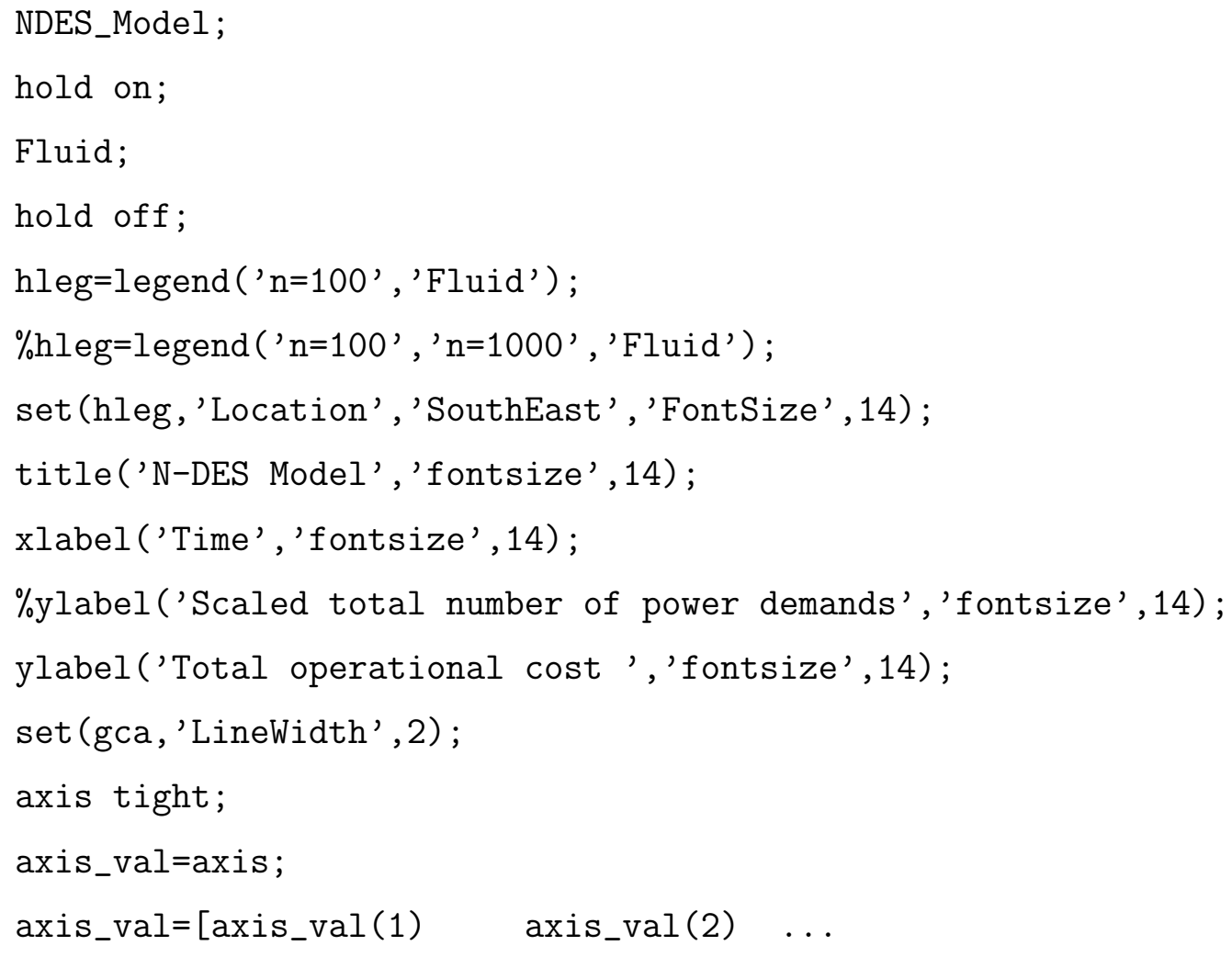


axis_val (3)*1.1 axis_val (4)*1.1];

$\operatorname{axis}($ axis_val);

\section{B.4 Simulation Programs of RES Model}

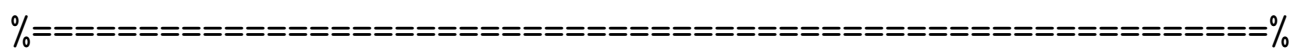

$\%$ This is the RES model simulation program. In this example\%

$\%$ the optimal policy is tested where the total number of $\%$ $\%$ power demands that can be served by batteries is fixed to\% $\%$ h_star. It is assumed that this is an initially empty sy-\% $\%$ stem. Power demands arrive according to Poisson process \% $\%$ with very big rate Lamda and are served by batteries and \% $\%$ grid with exponentially distributed times of means $1 / M_{-} b \%$ $\%$ and $1 / M_{-} g$ respectively. There are infinite number of ser $-\%$ $\%$ vers, $N$, in total and empty battery are assumed to be re- $\%$ $\%$ charged from grid with exponential time with mean $1 /$ chg. $\%$ $\%$ The observation time is $[0,10.1]$. X2 is the scaled total \% $\%$ number of power demands in the system. P2_star is the op-\% $\%$ timal total operational cost given the optimal policy. $\%$

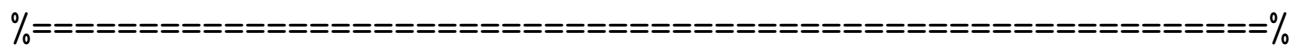
\%function Run(n,color)

$\mathrm{T}=10.1$;

$\mathrm{N}=2200 ; \%$ Number of servers/Grid capacity.

$\mathrm{k} 1=2$;

$\mathrm{k} 2=1.5$;

M_g=2.5; \% Service rate from grid.

M_b=0.5; \% Service rate from battery.

alpha $=2$; $\%$ Battery recharging rate from grid.

$\mathrm{n}=1000$;

Lamda $=2 * n ; \%$ Arrival rate.

h_star $=(1 / 2) *\left(\left(\mathrm{k} 1 * M_{-} \mathrm{b} / \mathrm{M}_{-} \mathrm{g}\right)-\left(\mathrm{k} 2 * \mathrm{M}_{-} \mathrm{b} / \mathrm{al} \mathrm{pha}\right)\right)$ 


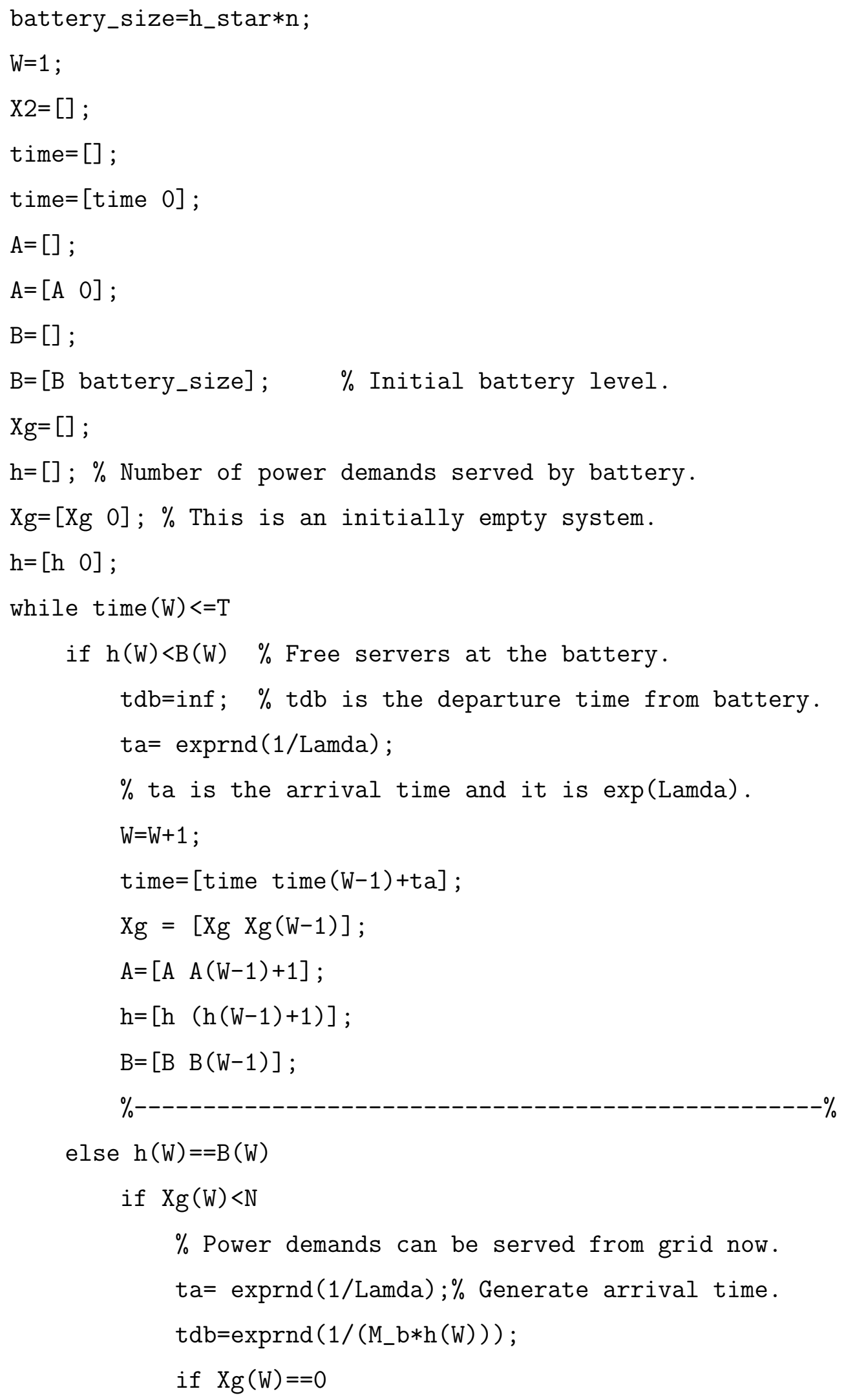




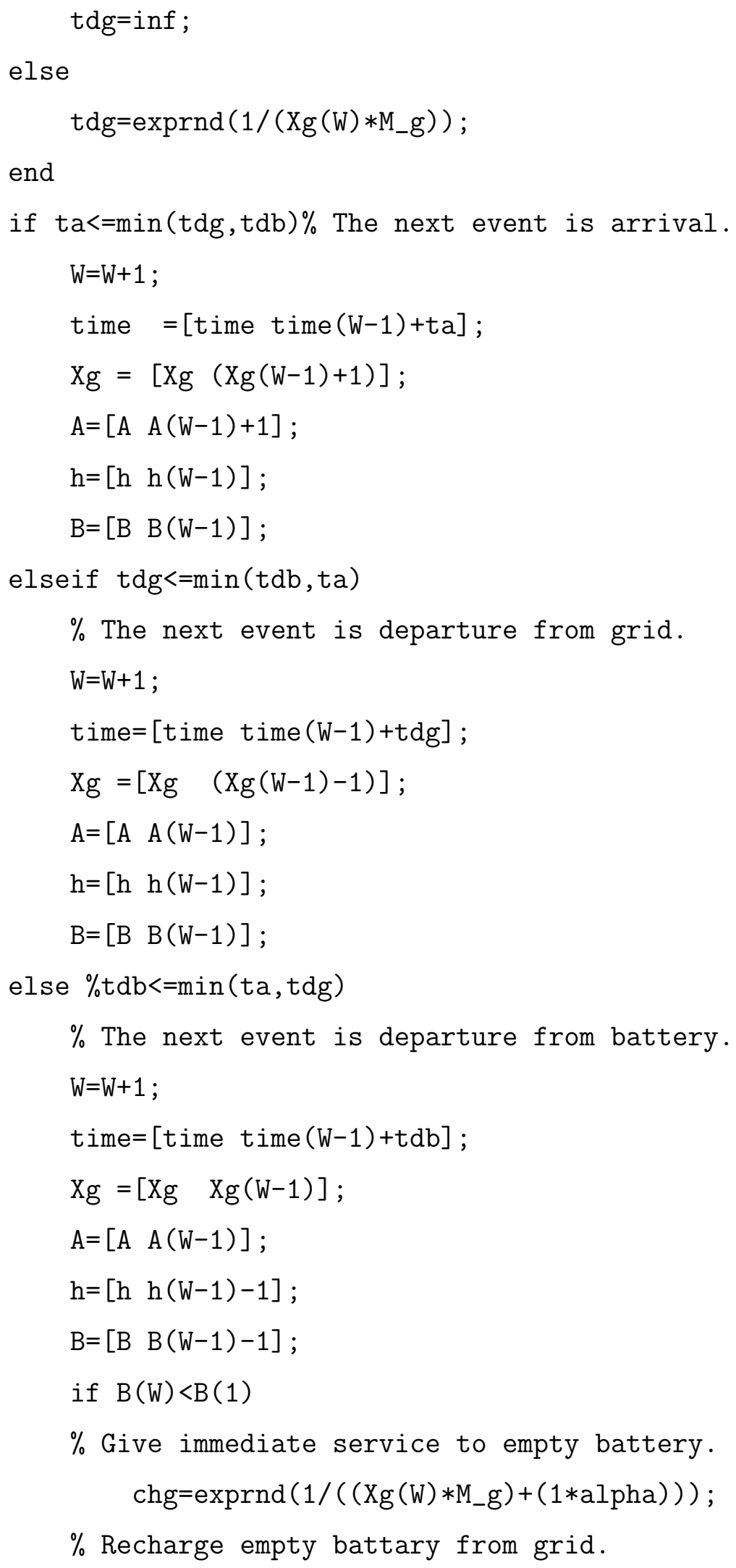




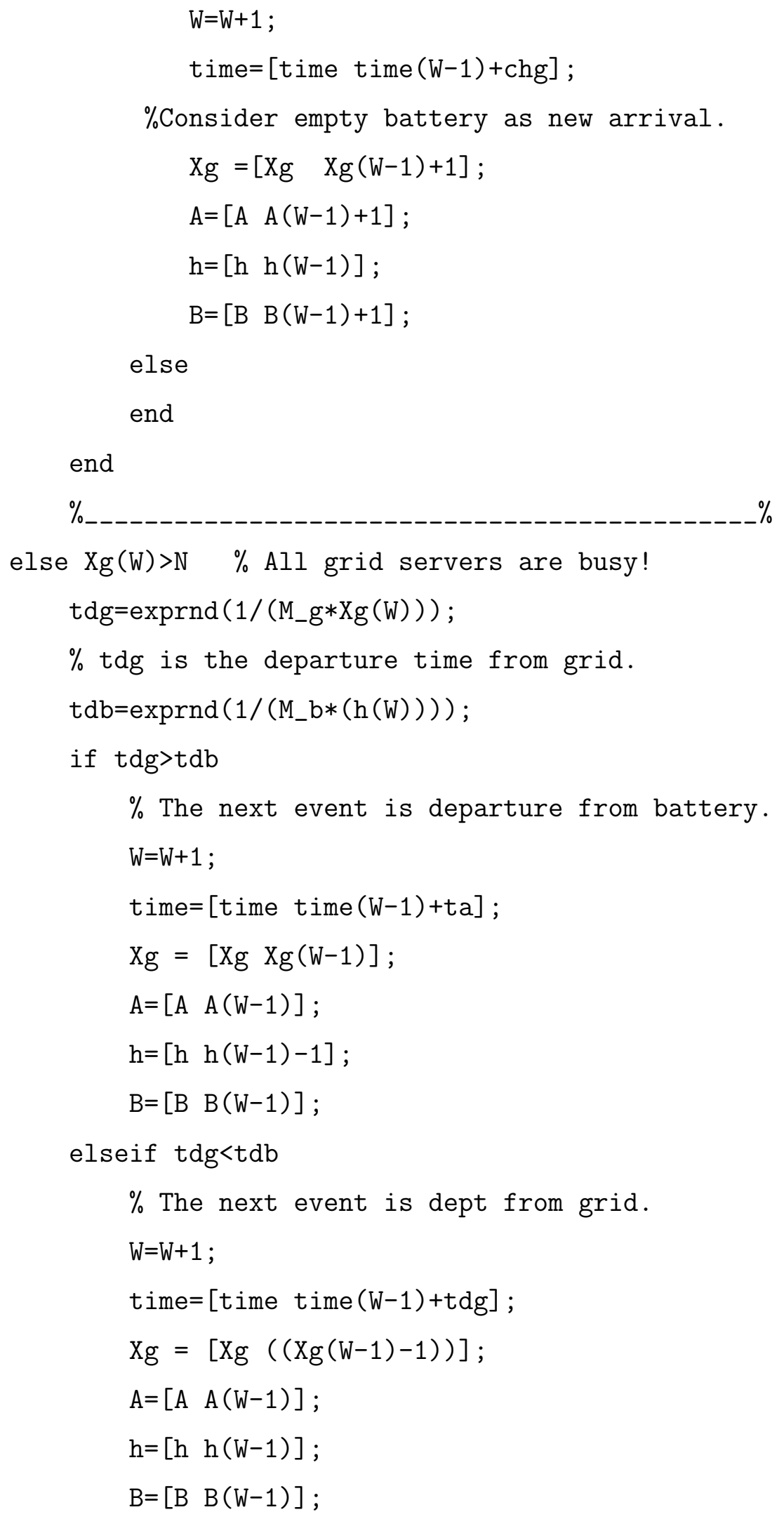


end

end

end

end

$j=1$;

h_intc $=[0]$;

h_bar $=(\mathrm{h} . / \mathrm{n}) \cdot{ }^{\wedge} 2$;

for $j=2:$ size (time, 2$)$

$h_{-}$intc $=\left[h_{-}\right.$intc $\quad h_{-}$intc $(j-1)+(\operatorname{time}(j)-\operatorname{time}(j-1)) \ldots$

*h_bar $(j-1)]$;

end

$j=1$;

$\mathrm{Xg}$-int2 $=[0]$;

$\mathrm{Xg}$ _bar $=\mathrm{Xg} \cdot / \mathrm{n}$;

for $j=2:$ size (time, 2)

$$
\begin{aligned}
& \text { Xg_int2 }=\left[X_{\text {g__int2 }} \mathrm{Xg}_{-} \text {int2 }(j-1)+(\operatorname{time}(j)-\operatorname{time}(j-1)) \ldots\right. \\
& \text { *(Xg_bar }(j-1))] \text {; }
\end{aligned}
$$

end

$\mathrm{X} 2=(\mathrm{Xg}+\mathrm{h}) \cdot / \mathrm{n}$;

plot (time, X2, 'LineWidth', 2)

\%plot (time, X2, 'Color' , color , 'LineWidth',2)

P2_star $=[]$;

\%P2_star $=\mathrm{k} 1 *\left(\mathrm{Xg}_{\text {_int }}\right.$./time $)+\left(\mathrm{k} 2 *\left(\mathrm{M}_{-} \mathrm{b} / \mathrm{al}\right.\right.$ pha $) * \mathrm{~h} \_$star $) .$.

+ h_intc./time;

lower_bound $=\mathrm{k} 1 *\left(\right.$ Lamda $\left./ M_{-} g\right)-\left(\mathrm{k} 1 *\left(M_{-}\right.\right.$b $\left./ M_{-} g\right) * h_{-}$star $) \ldots$ $+\left(\mathrm{k} 2 *(\right.$ M_b/alpha $) * \mathrm{~h}_{-}$star $)+\mathrm{h} \_$star ${ }^{\wedge} 2$

$\%$ plot (time, P2_star, '--g', 'LineWidth' ,2)

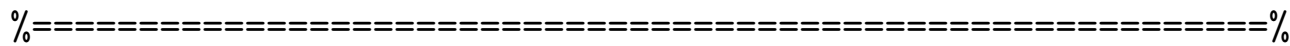

$\%$ This program creates plots for the deterministic RES mod- $\%$ $\%$ el. X2 is the total number of power demands in the $\%$ 


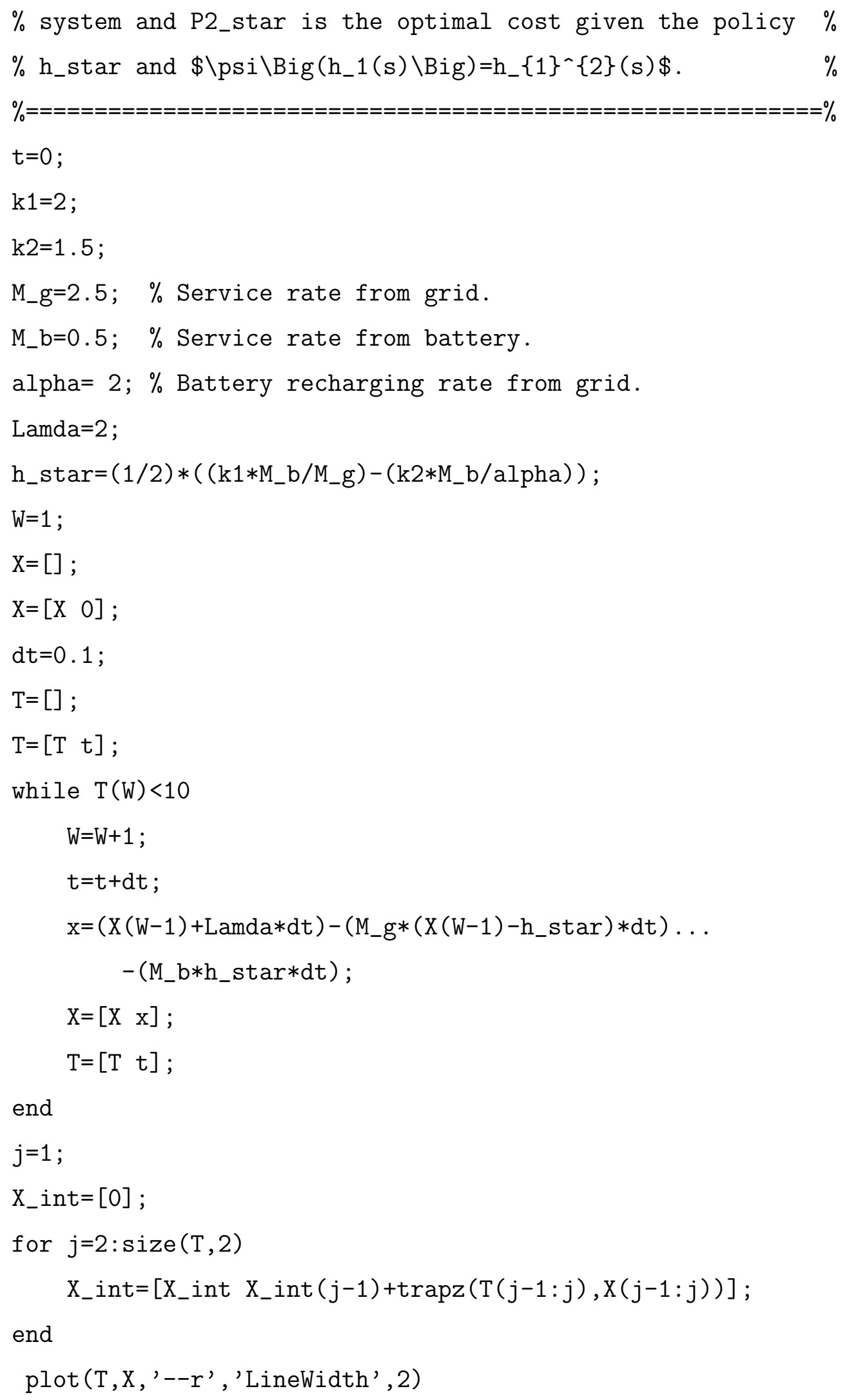




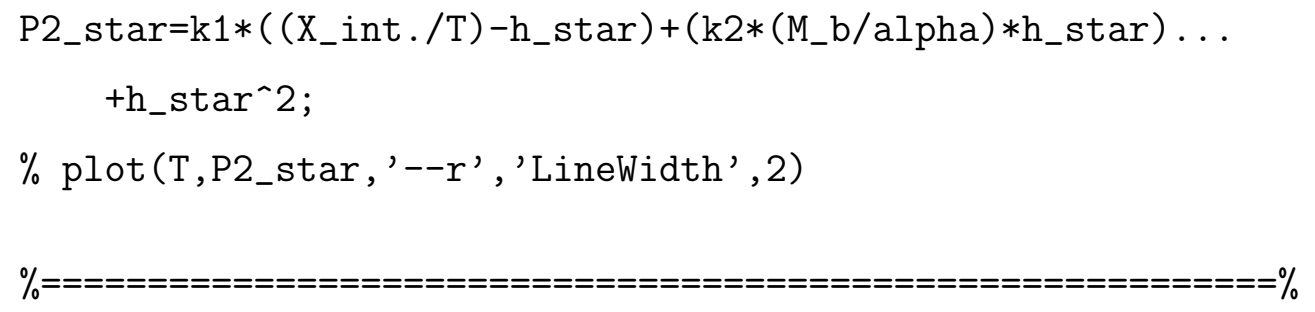

